TRANSACTIONS OF THE

AMERICAN MATHEMATICAL SOCIETY

Volume 363, Number 6, June 2011, Pages 2865-2890

S 0002-9947(2011)05272-4

Article electronically published on January 7, 2011

\title{
LIFTING GROUP ACTIONS AND NONNEGATIVE CURVATURE
}

\author{
KARSTEN GROVE AND WOLFGANG ZILLER
}

\begin{abstract}
We examine the question when a group acting by cohomogeneity one on the base of a principal G-bundle can be lifted to the total space and commutes with the action by $\mathrm{G}$. We answer this question completely when the base of the principle bundle is 4-dimensional.
\end{abstract}

Since the emergence of the fundamental structure theorem for complete open manifolds of nonnegative curvature due to Cheeger and Gromoll [CG], one of the central issues in this area has been to what extent the converse to this so-called soul theorem holds. In other words: Which total spaces of vector bundles over compact nonnegatively curved manifolds admit (complete) metrics with nonnegative curvature? The first examples where no such metrics exist were found by Özaydin and Walschap OW]. More recently a wealth of other examples have been found by Belegradek and Kapovitch BK1, BK2. So far, however, no obstructions are known when the base has finite fundamental group, and in particular when it is simply connected.

It is well known and easy to see that all vector bundles over compact simply connected 2- and 3-manifolds with nonnegative curvature (i.e. $\mathbb{S}^{2}$ and $\mathbb{S}^{3}$ by [Ha] admit complete metrics with nonnegative curvature. The first nontrivial case was treated in [GZ, where it was shown that all vector bundles over $\mathbb{S}^{4}$ can be equipped with such a metric. In this paper, we consider vector bundles over the remaining known closed simply connected 4-manifolds with nonnegative curvature, i.e., $\mathbb{C P}^{2}$, $\mathbb{S}^{2} \times \mathbb{S}^{2}$, and $\mathbb{C} \mathbb{P}^{2} \# \pm \mathbb{C P}^{2}$. One easily sees that a vector bundle over any of these spaces admits a complete metric of nonnegative curvature if its structure group reduces to a torus; see Theorem 4.5. It turns out that most vector bundles over $\mathbb{S}^{2} \times \mathbb{S}^{2}$ and $\mathbb{C P}^{2} \# \pm \mathbb{C P}^{2}$ are of this form; see Theorem 4.3. On the other hand, for vector bundles over $\mathbb{C P}^{2}$, the structure group almost never reduces to a torus. As a consequence of one of our main results we have:

Theorem 1. The total space of every vector bundle over $\mathbb{C P}^{2}$ with nontrivial second Stiefel Whitney class $w_{2}$ admits a complete metric of nonnegative sectional curvature.

In the case of $w_{2}=0$, our methods will show that half of all 3-dimensional vector bundles, those with $p_{1} \equiv 0 \bmod 8$, admit nonnegative curvature, and they all do when the fiber dimension is at least 5 . We do not know if all of the remaining

Received by the editors November 15, 2008

2010 Mathematics Subject Classification. Primary 53C29, 53 C07.

The first author was supported in part by the Danish Research Council.

The second author was supported by the Francis J. Carey Term Chair and the Clay Institute. Both authors were supported by grants from the National Science Foundation. 
bundles with $w_{2}=0$ admit nonnegative curvature, although some of them do since their structure group reduces to a torus.

In the more special case of complex vector bundles, we will see that:

Theorem 2. The total space of any complex rank 2 vector bundle over $\mathbb{C P}^{2}$ admits a complete metric of nonnegative curvature if its first Chern class $c_{1}$ is odd. The same is true if $c_{1}$ is even and the discriminant $\Delta=c_{1}^{2}-4 c_{2}$ satisfies $\Delta \equiv 0 \bmod 8$.

When $c_{1}$ is even, this is again half of all possible complex vector bundles since in general $\Delta \equiv 0 \bmod 4$. Although we do not know an explicit connection, it is tantalizing to observe that in the classification of (stable) holomorphic vector bundles over $\mathbb{C P}^{2}$, see [OSS, one also has the same division into $c_{1}$ even and odd ( $c_{1}$ odd being easier) and that the parity of $\Delta / 4$ in the case $c_{1}$ even is equally important.

In $\left[\mathrm{Ri}\right.$, it was shown that stably every vector bundle over $\mathbb{S}^{n}$ admits a complete metric of nonnegative curvature. Our analysis yields the same claim for vector bundles over each of the known simply connected 4-manifolds with nonnegative curvature; in fact,

Corollary. The total space of any vector bundle of rank $k \geq 6$ over $\mathbb{C P}^{2}, \mathbb{S}^{2} \times \mathbb{S}^{2}$, or $\mathbb{C P}^{2} \#-\mathbb{C P}^{2}$ admits a complete metric with nonnegative curvature.

Our results rely on constructing invariant metrics of nonnegative curvature on principal bundles, and then obtaining the desired metrics on the associated bundles from the well-known curvature increasing property of Riemannian submersions. This of course also implies that the associated sphere bundles over $\mathbb{C P}^{2}$ admit nonnegative curvature as well, giving rise to an interesting new class of compact examples with nonnegative curvature. The topology of these compact manifolds has recently been studied in [EZ].

In general it is a very difficult problem to decide which principal bundles over nonnegatively curved manifolds admit metrics with nonnegative curvature. A general construction of principal bundles over manifolds of cohomogeneity one, i.e. G-manifolds with one-dimensional orbit space, was found in GZ] (cf. section 1). There it was also shown that a large class of cohomogeneity one manifolds, the ones where the singular orbits have codimension two, carry metrics of nonnegative curvature, giving rise to such metrics on all principal bundles over $\mathbb{S}^{4}$.

Our point of departure here is that, as in the case of $\mathbb{S}^{4}$, each of the manifolds $\mathbb{C P}^{2}, \mathbb{S}^{2} \times \mathbb{S}^{2}$, and $\mathbb{C P}^{2} \#-\mathbb{C P}^{2}$ support cohomogeneity one actions with singular orbits of codimension two. Therefore so do all the principal bundles constructed by the cohomogeneity one method alluded to above. It remains to determine which bundles one gets this way, a topological problem which is considerably more involved than the corresponding one for bundles over $\mathbb{S}^{4}$ solved in GZ.

One can formulate this problem more generally in purely topological terms as follows:

Problem. Given a principal L-bundle $P \rightarrow M$ over a G-manifold $M$, when does the action of $\mathrm{G}$ on $M$ lift to an action of $\mathrm{G}$, or possibly a cover of $\mathrm{G}$, on the total space $P$, such that the lift commutes with L?

We will refer to such a lift as a commuting lift. This problem has been studied extensively; see e.g. $[\mathrm{HH}],[\mathrm{HY},[\mathrm{La},[\mathrm{PS},[\mathrm{St}],[\mathrm{TD}]$ and the references therein. However, apart from the general result $[\mathrm{PS}]$ that every action of a semisimple group 
admits a commuting lift to the total space of every principal circle or more generally torus bundle, the results seem to be difficult to apply in concrete cases.

In the case where the action on the base is cohomogeneity one, one has the cohomogeneity one method alluded to above. If the action has a lift to the total space, it forms, together with the action of L, another cohomogeneity one action, and it is a straightforward exercise to classify all possible group diagrams that the total space can have (see also $[\mathrm{HH}]$ ) . But it is now a highly nontrivial problem to determine which principal bundles one gets by this method. This in fact is the main contribution of our work presented here.

With this terminology we showed in GZ that the well-known cohomogeneity one action of $\mathrm{SO}(3)$ on $\mathbb{S}^{4}$, given by conjugation on the traceless symmetric $3 \times 3$ matrices, admits a commuting lift to every principal $\mathrm{SO}(k)$ bundle over $\mathbb{S}^{4}$. In contrast, we will show that the cohomogeneity one action of $\mathrm{SO}(3)$ on $\mathbb{C P}^{2}$ does not lift to every principal bundle over $\mathbb{C P}^{2}$, giving rise to the exceptions in Theorems $\mathrm{A}$ and B. More precisely, in this language, the topological main result behind Theorems $\mathrm{A}$ and $\mathrm{B}$ can be formulated as:

Theorem 3. Let $P \rightarrow \mathbb{C P}^{2}$ be a principal $\mathrm{SO}(3)$-bundle. The cohomogeneity one $\mathrm{SO}(3)$ subaction of the standard $\mathrm{SU}(3)$-action on $\mathbb{C P}^{2}$ admits a commuting lift to $P$ if and only if one of the following holds:

a) The principal bundle is not spin, i.e. $w_{2}(P) \neq 0$.

b) The bundle is spin and $p_{1}(P) \equiv 0 \bmod 8$.

c) The Pontryagin class satisfies $p_{1}(P)=4 r^{2}$ for some integer $r>0$.

More generally, our methods address the question which principal $\mathrm{SO}(k)$ bundles over each simply connected cohomogeneity one 4-manifold admit a commuting lift. It will follow, e.g., that the above $\mathrm{SO}(3)$ action lifts to every principal $\mathrm{SO}(k)$ bundle with $k \geq 5$. We will be able to answer this question almost completely. There is only one $\mathbb{Z}_{2}$ ambiguity left as to whether the cohomogeneity action by $\mathrm{SU}(2)$ on $\mathbb{C P}^{2}$ which has a fixed point lifts to an $\mathrm{SO}(k)$ principal bundle with $k \geq 5$; see Theorem 5.3. We will see that, in general, the lifting problem for $\mathrm{SO}(4)$ bundles can be reduced to $\mathrm{SO}(3)$ bundles (see section one).

We should mention that the manifold $\mathbb{C P}^{2} \# \mathbb{C P}^{2}$, according to $\mathrm{Pa}$ ] (cf. Ho]), does not admit any cohomogeneity one action and hence the methods in this paper will not apply in this case.

The paper is organized as follows. In section one we briefly recall the basic properties of cohomogeneity one manifolds needed in our paper, the principal bundle construction, and its characterization in terms of the existence of commuting lifts. In section two, we describe the cohomogeneity one actions on simply connected four manifolds. Section three is devoted to the topological classification of principal $\mathrm{SO}(k)$ bundles over 4-manifolds in terms of invariants computable in our context. In sections four and five we derive which principal $\mathrm{SO}(k)$ bundles over any given 1-connected, 4-dimensional cohomogeneity one manifold admit a commuting lift. Specifically, section four is devoted to the classification over cohomogeneity one manifolds with singular orbits of codimension two needed for the geometric consequences of the paper, and section five deals with the classification for cohomogeneity one actions where at most one orbit has codimension two. 
It is our pleasure to thank V. Kapovitch, N. Kitchloo, I. Madsen, J. Shaneson, and B. Wilking for helpful discussions. This work was completed while the second author was visiting IMPA and he would like to thank the Institute for its hospitality.

\section{BASIC SETUP}

Throughout the paper, we will make extensive use of the structure of cohomogeneity one manifolds, which we briefly recall here for convenience. For more details we refer to e.g. $\mathrm{AA}, \mathrm{Br}, \mathrm{GZ}, \mathrm{Mo}$.

A connected manifold $M$ is said to have cohomogeneity one if it supports a smooth action by a compact Lie group $\mathrm{G}$, such that the orbit space $M / \mathrm{G}$ is onedimensional. Here we are only interested in the case where $M$ is compact and simply connected, and $\mathrm{G}$ is connected. In this case $M / \mathrm{G}$ is an interval, and the nonprincipal orbits are singular (of codimension at least two) and correspond exactly to the end points of $M / \mathrm{G}$.

Fix an auxiliary G-invariant metric on $M$, such that $M / \mathrm{G}=[-1,1]$ isometrically, and let $c$ be a geodesic perpendicular to all orbits. We denote by $\mathrm{H}$ the principal isotropy group $\mathrm{G}_{c(0)}$ at $c(0)$, which is equal to the isotropy groups $\mathrm{G}_{c(t)}$ for all $t \neq \pm 1$, and by $\mathrm{K}^{ \pm}$the isotropy groups at $c( \pm 1)=x_{ \pm}$. In terms of this we have

$$
M=\mathrm{G} \times \mathrm{K}^{-} \mathbb{D}^{-} \cup \mathrm{G} \times \mathrm{K}^{+} \mathbb{D}^{+}=M_{-} \cup M_{+}
$$

where $\mathbb{D}^{ \pm}$denotes the normal disc to the orbit $G x_{ \pm}=\mathrm{G} / \mathrm{K}^{ \pm}=B_{ \pm}$at $x_{ \pm}$, and the gluing is done along $M_{0}=M_{-} \cap M_{+}=\mathrm{G} / \mathrm{H}$ with the identity map. It is important to note that $\mathbb{S}^{ \pm}=\partial \mathbb{D}^{ \pm}=\mathrm{K}^{ \pm} / \mathrm{H}$, and that the diagram of groups

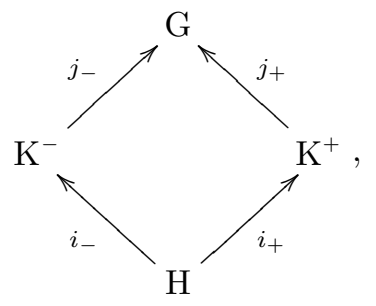

which we also record as $\mathrm{H} \subset\left\{\mathrm{K}^{-}, \mathrm{K}^{+}\right\} \subset \mathrm{G}$, determines $M$. Conversely, such a group diagram with $\mathrm{K}^{ \pm} / \mathrm{H}=\mathbb{S}^{l_{ \pm}}$defines a cohomogeneity one G-manifold, given by (1.1). The action of $\mathrm{G}$ on $M$ is given by left multiplication in the first component on each half, and one easily checks that this action has isotropy groups as in (1.2).

Notice though that the description of $M$ by a group diagram depends on the choice of a $\mathrm{G}$ invariant metric. The description of all group diagrams coming from a different choice of a metric, or equivalently the equivariant diffeomorphism classification of such $\mathrm{G}$ manifolds, is given by (cf. [GWZ], $[\mathrm{Ne},[\mathrm{Br}]$ ):

Lemma 1.3. If $\mathrm{H} \subset\left\{\mathrm{K}^{-}, \mathrm{K}^{+}\right\} \subset \mathrm{G}$ defines a cohomogeneity one manifold, then the only cohomogeneity one $\mathrm{G}$-manifolds which are equivariantly diffeomorphic to it are of the form $a \mathrm{H} a^{-1} \subset\left\{a \mathrm{~K}^{-} a^{-1}, n a \mathrm{~K}^{+}(n a)^{-1}\right\} \subset \mathrm{G}$ for some $a \in \mathrm{G}$ and $n \in \mathrm{N}(\mathrm{H})_{0}$.

Next we recall from GZ that the above characterization of cohomogeneity one manifolds also allows for a natural construction of principal bundles within this category. 
Let $\mathrm{L}$ be any compact Lie group, and $M$ a cohomogeneity one manifold with group diagram $\mathrm{H} \subset\left\{\mathrm{K}^{-}, \mathrm{K}^{+}\right\} \subset \mathrm{G}$, where $\mathrm{G}$ is allowed to act ineffectively.

For any Lie group homomorphisms $\phi_{ \pm}: \mathrm{K}^{ \pm} \rightarrow \mathrm{L}$ with $\phi_{+} \circ i_{+}=\phi_{-} \circ i_{-}=\phi_{0}$, let $P$ be the cohomogeneity one $\mathrm{L} \times \mathrm{G}$-manifold with diagram

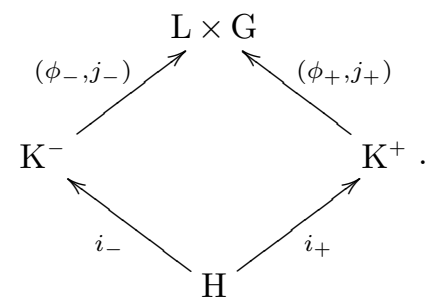

The crucial property of our cohomogeneity construction is the following characterization:

Lemma 1.5 (Principal Bundle Lemma). Let $M$ be the cohomogeneity one Gmanifold defined by (1.2).

(a) If $P$ is a cohomogeneity one manifold defined by (1.4), then $\mathrm{L}$ acts freely on $P$, and the quotient $P / \mathrm{L}$ with its induced action by $\mathrm{G}$ is equivariantly diffeomorphic to $M$.

(b) Conversely, a principal L-bundle $\pi: P \rightarrow M$ admits a lift by $\mathrm{G}$, or possibly a cover of $\mathrm{G}$, that commutes with $\mathrm{L}$ if and only if $P$ can be described as a cohomogeneity one $\mathrm{L} \times \mathrm{G}$-manifold defined as in (1.4).

Proof. First observe that for a left action of $\mathrm{L} \subset \mathrm{G}$ on any homogeneous space $\mathrm{G} / \mathrm{H}$, the isotropy groups are given by $\mathrm{L}_{g \mathrm{H}}=\mathrm{L} \cap g \mathrm{H}^{-1}$, and thus if $\mathrm{L}$ is normal in $\mathrm{G}$, it acts freely if and only if $\mathrm{L} \cap \mathrm{H}=\{e\}$. Applying this to each $\mathrm{L} \times \mathrm{G}$ orbit in $P$ shows that $\mathrm{L}$ acts freely on $P$ since the embeddings $\left(\phi_{ \pm}, j_{ \pm}\right)$are injective in the second component. Since $\mathrm{G}$ is also a normal subgroup, it induces an action on the quotient $P / \mathrm{L}$. Let $c(t), t \in[-1,1]$ be a geodesic in an $\mathrm{L} \times \mathrm{G}$ invariant metric on $P$ normal to all $\mathrm{L} \times \mathrm{G}$ orbits, and with isotropy groups as in (1.4). Then $c$ is a horizontal geodesic under the projection $\pi: P \rightarrow P / \mathrm{L}$, and hence $\pi \circ c(t)$ is a geodesic in $M$ normal to all $\mathrm{G}$ orbits. Furthermore, $\pi$ is $\mathrm{G}$ equivariant and since $\mathrm{L}$ acts transitively on the fibers of $\pi$, it follows that $g \pi(c(-1))=\pi(c(-1))$ if and only if there exists an $\ell \in \mathrm{L}$ with $(\ell, g) c(-1)=c(-1)$ and thus $(\ell, g)=\left(\phi_{-}(k), j_{-}(k)\right)$ for some $k \in \mathrm{K}^{-}$. Similarly for $c(1)$ and $c(0)$, which implies that (1.2) is the group diagram for the $\mathrm{G}$ action on $P / \mathrm{L}$ and hence $P / \mathrm{L}$ is G-equivariantly diffeomorphic to $M$.

To prove (b), assume there exists a cover $\sigma: \tilde{\mathrm{G}} \rightarrow \mathrm{G}$ and an $\mathrm{L} \times \tilde{\mathrm{G}}$ action on $P$ such that $g \cdot p=\sigma(g) \cdot \pi(p)$ for all $g \in \tilde{\mathrm{G}}$ and $p \in P$. Since $P /(\mathrm{L} \times \tilde{\mathrm{G}})=M / \mathrm{G}$, the action of $\mathrm{L} \times \tilde{\mathrm{G}}$ is cohomogeneity one. We can define an ineffective action by $\tilde{\mathrm{G}}$ on $M$ with isotropy groups $\tilde{\mathrm{K}}^{ \pm}=\sigma^{-1}\left(\mathrm{~K}^{ \pm}\right)$and $\tilde{\mathrm{H}}=\sigma^{-1}(\mathrm{H})$ and embeddings $\tilde{j}_{ \pm}$. For simplicity, we denote this action again by $\mathrm{G}$ with $\sigma=\mathrm{Id}$. To determine its group diagram, choose a metric on $P$ such that $\mathrm{L} \times \mathrm{G}$ acts isometrically and such that the induced metric on $M$, which makes $\pi$ into a Riemannian submersion, coincides with the given metric on $M$. If we let $\tilde{c}$ be a horizontal lift of the geodesic $c$ in $M$ normal to all $\mathrm{G}$ orbits, it follows that $\tilde{c}$ is normal to all $\mathrm{L} \times \mathrm{G}$ orbits as well. Furthermore, $(\ell, g) \tilde{c}(-1)=\tilde{c}(-1)$ implies that $g c(-1)=c(-1)$ and thus $g=j_{-}(k)$ for some $k \in \mathrm{K}^{-}$. The element $\ell$ is uniquely determined by $k$ since $\mathrm{L}$ acts freely. 
Letting $\ell=\phi_{-}(k)$, it follows that $\phi_{-}$is a homomorphism. Hence the lift $\mathrm{L} \times \mathrm{G}$ has a group diagram as in (1.4).

In order to avoid having to consider covers of $G$ as in Lemma 1.5 (b), we will assume from now on that the action of $\mathrm{G}$ on $M$ is an almost effective action by a simply connected group, possibly a product with a torus. This will ensure that we obtain all possible lifts of the original action.

Notice also that in the group diagram (1.4), the action of $\mathrm{L} \times \mathrm{G}$ may not be effective, even if the action of $G$ is, in particular the lift may not be a product group.

We now collect some useful properties of these commuting lifts:

Lemma 1.6. Assume that $\pi: P \rightarrow M$ is an $\mathrm{L}$ principal bundle and that $\mathrm{G}$ acts on $M$.

(a) If $\mathrm{G}$ admits a commuting lift to a reduction $P^{*}$ of $P$ corresponding to a subgroup $\mathrm{L}^{*} \subset \mathrm{L}$ it admits a commuting lift to $P=P^{*} \times \mathrm{L}^{*} \mathrm{~L}$.

(b) If $\tilde{\mathrm{L}}$ is a finite cover of $\mathrm{L}$ and $P$ admits a cover $\tilde{P}$ yielding a corresponding principal $\tilde{\mathrm{L}}$ bundle $\tilde{P} \rightarrow M$, then $\tilde{P}$ admits a commuting lift if and only if $P$ does.

(c) If $\mathrm{L}$ is a local product $\mathrm{L}_{1} \cdot \mathrm{L}_{2}$, then $\mathrm{G}$ admits a commuting lift to $P$ if and only if it admits a commuting lift to the principal $\mathrm{L}_{1}$ bundle $P_{1}=P / \mathrm{L}_{2}$ and the principal $\mathrm{L}_{2}$ bundle $P_{2}=P / \mathrm{L}_{1}$.

Proof. The claims in (a) and (b) are easily verified. In one direction, (c) follows immediately since if $\mathrm{G}$ admits a lift to $P$ it also admits a lift to $P / \mathrm{L}^{\prime}$ for any normal subgroup $\mathrm{L}^{\prime}$ of $\mathrm{L}$. To see the converse, we first reduce to the case of a product group $\mathrm{L}=\mathrm{L}_{1} \times \mathrm{L}_{2}$, by applying (b) to the principal $\left(\mathrm{L}_{1} / F\right) \times\left(\mathrm{L}_{2} / F\right)$ bundle $P / F$, where $F=\mathrm{L}_{1} \cap \mathrm{L}_{2}$ is a finite normal subgroup of $\mathrm{L}_{i}$. In the case of a product group, the $\mathrm{L}=\mathrm{L}_{1} \times \mathrm{L}_{2}$ principal bundle $P$ has classifying map given by $f=\left(f_{1}, f_{2}\right): M \rightarrow B_{\mathrm{L}_{1}} \times B_{\mathrm{L}_{2}}$ where the $f_{i}$ are the classifying maps of $P_{i}$. Hence the $\mathrm{L}$ principal bundle $P$ is determined up to isomorphism by the $\mathrm{L}_{i}$ principal bundles $P_{i}$. Now consider $\tilde{P}=\left\{\left(x_{1}, x_{2}\right) \in P_{1} \times P_{2} \mid \pi_{1}\left(x_{1}\right)=\pi_{2}\left(x_{2}\right)\right\}$. $\tilde{P}$ is clearly a principal $\mathrm{L}_{1} \times \mathrm{L}_{2}$ bundle over $M$ and since $\tilde{P} / \mathrm{L}_{i}=P_{i}$ the bundle $\tilde{P}$ must be isomorphic to $P$. If $P_{i}$ now admits a lift that commutes with $\mathrm{L}_{i}$, it clearly also admits a lift to $\tilde{P}$ that commutes with $\mathrm{L}_{1} \times \mathrm{L}_{2}$.

In the case of cohomogeneity one actions we have:

Lemma 1.7. Let $(M, \mathrm{G})$ be a cohomogeneity one manifold as in (1.2) and let $(P, \mathrm{~L} \times \mathrm{G})$ be an $\mathrm{L}$ principal bundle over $M$ as in (1.4). Then we have:

(a) If a subgroup $\mathrm{L}^{*} \subset \mathrm{L}$ contains the image groups $\phi_{ \pm}\left(\mathrm{K}^{ \pm}\right)$, then $P$ admits a reduction to $\mathrm{L}^{*}$.

(b) Suppose $\mathrm{G}$ is a local product $\mathrm{G}_{1} \cdot \mathrm{G}_{2}$ and the subaction of $\mathrm{G}_{1} \times\{e\} \subset \mathrm{G}_{1} \cdot \mathrm{G}_{2}$ has the same orbits as the $\mathrm{G}$-action. Then the action of $\mathrm{G}_{1} \cdot \mathrm{G}_{2}$ admits a commuting lift to $P$ if and only if $\mathrm{G}_{1}$ does.

Proof. To see (a), consider the cohomogeneity one manifold $P^{*}$ defined by $\mathrm{H} \subset$ $\left\{\mathrm{K}^{-}, \mathrm{K}^{+}\right\} \subset \mathrm{L}^{*} \times \mathrm{G}$. Then $P=P^{*} \times \mathrm{L}^{*} \mathrm{~L}$, which one easily verifies by showing that the $\mathrm{L} \times \mathrm{G}$-actions on both have the same isotropy groups . Hence $P$ reduces to $P^{*}$.

In order to prove (b) we assume, by making the action almost effective if necessary, that $G_{1}$ and $G_{2}$ are simply connected and that $G=G_{1} \times G_{2}$. Suppose 
the $\mathrm{G}_{1}$-action on $M$ with diagram $\mathrm{H} \subset\left\{\mathrm{K}^{-}, \mathrm{K}^{+}\right\} \subset \mathrm{G}_{1}$ lifts. We first claim that the diagram for the extended almost effective $\mathrm{G}_{1} \times \mathrm{G}_{2}$-action on $M$ has isotropy groups $\mathrm{H} \times \mathrm{G}_{2} \subset\left\{\mathrm{K}^{-} \times \mathrm{G}_{2}, \mathrm{~K}^{+} \times \mathrm{G}_{2}\right\} \subset \mathrm{G}_{1} \times \mathrm{G}_{2}$, where the $\mathrm{G}_{2}$ factor is embedded diagonally in $\mathrm{G}_{1} \times \mathrm{G}_{2}$. Indeed, if $\mathrm{G}_{1} \times \mathrm{G}_{2} / \mathrm{S}$ is a homogeneous space where $\mathrm{G}_{1} \times\{e\} \subset \mathrm{G}_{1} \times \mathrm{G}_{2}$ acts transitively, then $\mathrm{S}$ projects onto the second factor in $\mathrm{G}_{1} \times \mathrm{G}_{2}$ and $\mathrm{G}_{1} \times \mathrm{G}_{2} / \mathrm{S}=\mathrm{G}_{1} /\left(\mathrm{G}_{1} \times\{e\}\right) \cap \mathrm{S}$. Thus $\mathrm{S}=\mathrm{S}_{1} \cdot \mathrm{S}_{2}$ with $\mathrm{S}_{1}=\left(\mathrm{G}_{1} \times\{e\}\right) \cap \mathrm{S}$ and $\mathrm{S}_{2}$ a complementary normal subgroup. Since $\mathrm{S}_{2}$ projects onto $G_{2}$ and $G_{2}$ is simply connected, it follows that $S_{2} \simeq G_{2}$. Furthermore, $\mathrm{S}_{1} \cap \mathrm{S}_{2}=\{e\}$ and thus $\mathrm{S}$ is the direct product $\mathrm{S}=\mathrm{S}_{1} \times \mathrm{S}_{2}$ with $\mathrm{S}_{2}$ embedded diagonally. We now apply this argument to each orbit.

The desired homomorphisms in the construction of the lift of the $\mathrm{G}_{1} \times \mathrm{G}_{2}$-action can thus be taken to be the projection to the first factor followed by the homomorphisms used in the construction of the $\mathrm{G}_{1}$ lift.

Lemma 1.6 (c) will be particularly useful for us in the case of $\mathrm{L}=\mathrm{SO}(4)$. In $\mathrm{SO}(4)$ there are two normal subgroups $\mathrm{S}_{-}^{3}, \mathrm{~S}_{+}^{3}$ isomorphic to $\mathrm{S}^{3}$, defined by left and right multiplication by unit quaternions on $\mathbb{H} \simeq \mathbb{R}^{4}$, and $\mathrm{SO}(4) / \mathrm{S}_{ \pm}^{3}$ is isomorphic to $\mathrm{SO}(3)$. Hence, if $\mathrm{SO}(4) \rightarrow P \rightarrow M$ is a principal $\mathrm{SO}(4)$ bundle, there are two associated principal $\mathrm{SO}(3)$ bundles $P^{ \pm}=P / \mathrm{S}_{\mp}^{3}$ over $M$. The relationship between these bundles can be described as follows:

Proposition 1.8. Let $M$ be a compact, simply connected n-dimensional manifold and $\pi: P \rightarrow M$ a principal $\mathrm{SO}(4)$ bundle with associated principal $\mathrm{SO}(3)$ bundles $\pi_{ \pm}: P^{ \pm} \rightarrow M$. The principal bundle $P$ is uniquely determined by the $\mathrm{SO}(3)$ bundles $P^{ \pm}$and $w_{2}(P)=w_{2}\left(P^{ \pm}\right)$. Conversely, if $P^{ \pm} \rightarrow M$ are two principal $\mathrm{SO}(3)$ bundles with $w_{2}\left(P^{-}\right)=w_{2}\left(P^{+}\right)$, then there exists a principal $\mathrm{SO}(4)$ bundle $P \rightarrow M$ which gives rise to $P^{ \pm}$.

Proof. To see that $w_{2}(P)=w_{2}\left(P^{ \pm}\right)$, consider the following commutative diagram of homotopy sequences:

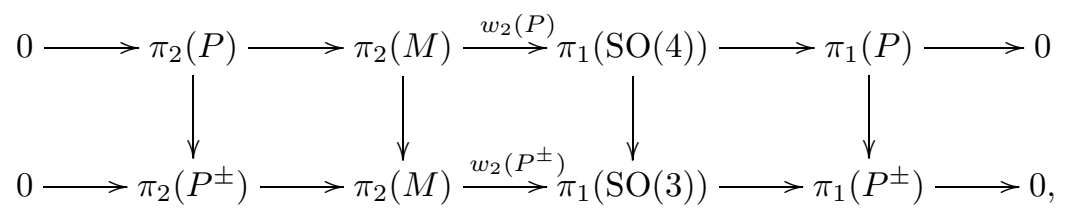

where the boundary map $\partial: \pi_{2}(M) \rightarrow \pi_{1}(\mathrm{SO}(k))=\mathbb{Z}_{2}$, if considered as an element of $\operatorname{Hom}\left(\pi_{2}(M), \mathbb{Z}_{2}\right)=H^{2}\left(M, \mathbb{Z}_{2}\right)$, is precisely $w_{2}$. (This follows, e.g., by observing that it is clearly true for the universal bundle.) Since $\pi_{1}(\mathrm{SO}(4)) \rightarrow \pi_{1}(\mathrm{SO}(3))$ is an isomorphism, it follows that $w_{2}(P)=w_{2}\left(P^{ \pm}\right)$. Furthermore, $P$ and $P^{ \pm}$are simply connected if and only if $w_{2} \neq 0$ and their fundamental group is $\mathbb{Z}_{2}$ if $w_{2}=0$.

Now assume that $w_{2}\left(P^{ \pm}\right)=0$. The bundles $P^{ \pm}$then admit (unique) two fold covers $\tilde{P}^{ \pm}$which are principal $\mathrm{S}^{3}$ bundles over $M$ classified by $f_{ \pm}: M \rightarrow B_{\mathrm{S}^{3}}$, and $f=\left(f_{-}, f_{+}\right): M \rightarrow B_{\mathrm{S}^{3}} \times \mathrm{S}^{3}$ defines a principal $\mathrm{S}^{3} \times \mathrm{S}^{3}$ bundle $\tilde{P}$ over $M$. The bundle $P=\tilde{P} /\{ \pm(1,1)\}$ is now the desired $\mathrm{SO}(4)$ principal bundle and uniqueness follows as well since $\pi_{1}(P)=\mathbb{Z}_{2}$.

If $w_{2}\left(P^{-}\right)=w_{2}\left(P^{+}\right) \neq 0$, we consider, as in the proof of Lemma 1.6. $\tilde{P}=$ $\left\{\left(x_{-}, x_{+}\right) \in P^{-} \times P^{+} \mid \pi_{-}\left(x_{-}\right)=\pi_{+}\left(x_{+}\right)\right\}$together with the natural principal $\mathrm{SO}(3)$ bundle projections $\sigma_{ \pm}: \tilde{P} \rightarrow P^{ \pm}$. $\tilde{P}$ is clearly also a principal $\mathrm{SO}(3) \times \mathrm{SO}(3)$ bundle over $M$. $\tilde{P}$ can be regarded as the pullback of the principal $\mathrm{SO}(3)$ bundle 
$P^{+} \rightarrow M$ via $\pi_{-}$, and also as the pullback of the principal SO(3) bundle $P^{-} \rightarrow M$ via $\pi_{+}$; i.e., we have the following commutative diagram of pullback bundles:

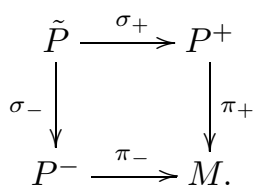

For both ways of looking at $\tilde{P}$, it follows that $w_{2}(\tilde{P})=0$ since the compositions $\pi_{2}\left(P^{ \pm}\right) \rightarrow \pi_{2}(M) \stackrel{w_{2}(P)}{\longrightarrow} \pi_{1}(\mathrm{SO}(3))$ are 0 , which implies that $0=\pi_{-}^{*}\left(w_{2}\left(P^{-}\right)\right)=$ $\pi_{-}^{*}\left(w_{2}\left(P^{+}\right)\right)=w_{2}(\tilde{P})$ and similarly for $\pi_{+}$. Furthermore, since $w_{2}\left(P^{ \pm}\right) \neq 0, P^{ \pm}$ are simply connected, and since $w_{2}(\tilde{P})=0$ we have $\pi_{1}(\tilde{P})=\mathbb{Z}_{2}$. Hence the unique two fold cover $P \rightarrow \tilde{P}$ is a spin cover of each bundle $\sigma_{ \pm}: \tilde{P} \rightarrow P^{ \pm}$. But $\mathrm{SO}(4)$ is the only two fold cover of $\mathrm{SO}(3) \times \mathrm{SO}(3)$ which is a two fold cover along each $\mathrm{SO}(3)$, and hence $P$ is a principal $\mathrm{SO}(4)$ bundle which clearly gives rise to $P^{ \pm}$in the original construction. Uniqueness follows from the same commutative diagram of principal bundles.

We furthermore remark that the principal $\mathrm{SO}(4)$ bundles whose structure group reduces to $\mathrm{U}(2)$, i.e. the complex vector bundles (resp. $\mathrm{SU}(2)$ ), are precisely those where either $P^{+}$or $P^{-}$reduces to an $\mathrm{SO}(2)$ bundle (resp. becomes trivial). The even more special $\mathrm{SO}(4)$ bundles where the structure group reduces to a 2 -torus, i.e. the direct sum of two complex line bundles, correspond to those where both $P^{+}$and $P^{-}$reduce to an $\mathrm{SO}(2)$ bundle. Finally, the bundles where the structure group reduces to $\mathrm{SO}(3)$ (i.e. the bundles with a section) correspond to the ones where $P^{+}$and $P^{-}$are isomorphic.

In terms of oriented vector bundles, the above relationship between $P$ and $P^{ \pm}$ can also be described as follows. If $E$ is the 4 dimensional vector bundle over $M$ with principal bundle $P$, then $\Lambda^{2}(E)=\Lambda_{-}^{2}(E) \oplus \Lambda_{+}^{2}(E)$ is given by the decomposition of a 2 form into its self dual and anti self dual parts. Then $\Lambda_{ \pm}^{2}(E)$ is the 3 dimensional vector bundle whose principal $\mathrm{SO}(3)$ bundle is $P^{ \pm}$, which follows from the fact that the decomposition $\mathfrak{s o}(4)=\Lambda^{2} \mathbb{R}^{4}=\Lambda_{-}^{2} \mathbb{R}^{4} \oplus \Lambda_{+}^{2} \mathbb{R}^{4}=\mathfrak{s o}(3) \oplus \mathfrak{s o}(3)$ is the decomposition of $\mathfrak{s o}(4)$ into simple ideals. Using this, one easily shows (cf. [DR]):

$$
p_{1}\left(P^{ \pm}\right)=p_{1}(P) \pm 2 e(P) .
$$

In the case of complex rank two bundles, one has the Chern classes $c_{1}$ and $c_{2}$ and since for the underlying real bundle $p_{1}(P)=c_{1}^{2}-2 c_{2}$ (cf. [Mi, 15.5]), the relationship (1.10) implies that:

$$
p_{1}\left(P^{+}\right)=c_{1}^{2} \text { and } p_{1}\left(P^{-}\right)=c_{1}^{2}-4 c_{2}
$$

and hence, under the usual embedding of $\mathrm{U}(2)$ in $\mathrm{SO}(4), P^{+}$is the one whose structure group reduces.

\section{Cohomogeneity One FOUR-MANifoldS}

According to Parker's classification $\mathrm{Pa}$ of all cohomogeneity one 4-manifolds, the only simply connected manifolds which admit such actions are $\mathbb{S}^{4}, \mathbb{C P}^{2}, \mathbb{S}^{2} \times$ $\mathbb{S}^{2}$, and $\mathbb{C P}^{2} \#-\mathbb{C P}^{2}$. We will analyze the lifting problem for each cohomogeneity one action by a connected compact group on these manifolds. 
In this section we describe the cohomogeneity one actions on simply connected 4-manifolds. Although these actions are exhibited in $\mathrm{Pa}$, we need to know the precise group picture for our applications. Recall that we can derive the group diagram (1.2) by choosing one singular orbit $B_{-}=\mathrm{G} / \mathrm{K}^{-}$with $\mathrm{K}^{-}=\mathrm{G}_{x_{-}}$and by choosing any geodesic $c(t)=\exp _{x_{-}}\left(t v_{-}\right)$with $v_{-}$normal to $B_{-}$since it will then automatically be normal to all orbits. We then need to determine the first $t_{0}$ when $c\left(t_{0}\right)$ meets $B_{+}$, i.e. when $\mathrm{G}_{c\left(t_{0}\right)}$ is bigger than the principal isotropy group H. Then $c\left(t_{0}\right)=x_{+}$and $\mathrm{K}^{+}=\mathrm{G}_{x_{+}}$and $\mathrm{H}=\mathrm{G}_{c(t)}, 0<t<t_{0}$.

We start with cohomogeneity one actions with singular orbits of codimension two, since they are the most important ones in geometric applications. In addition, with one exception, they are all cohomogeneity one under $\mathrm{G}=\mathrm{SO}(3)$ or $\mathrm{SU}(2)$ or an extension of an $\mathrm{SU}(2)$ cohomogeneity one action to $\mathrm{U}(2)$. We will describe them as an action with $\mathrm{G}=\mathrm{S}^{3}$ in order to obtain all possible lifts.

First recall that the linear $\mathrm{SO}(3)$ action on $\mathbb{S}^{4}$ corresponding to a maximal subgroup of $\mathrm{SO}(5)$, which played a pivotal role in $\mathrm{GZ}$, has the following group diagrams when lifted to $\mathrm{S}^{3}$ :

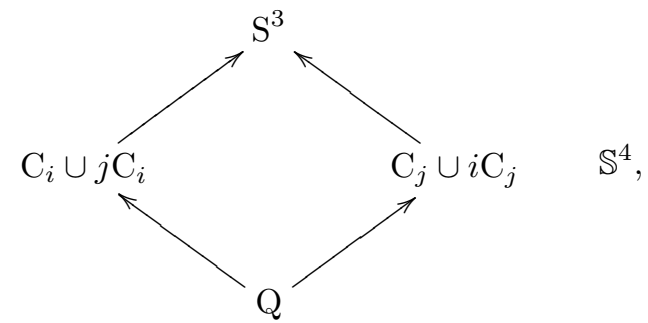

where $\mathrm{C}_{i}=\left\{e^{i \theta} \mid \theta \in \mathbb{R}\right\}, \mathrm{C}_{j}=\left\{e^{j \theta} \mid \theta \in \mathbb{R}\right\}$ are "coordinate" circle groups, and $\mathrm{Q}=\{ \pm 1, \pm i, \pm j, \pm k\}$ is the quaternion group.

Next consider $\mathbb{C P}^{2}$, where we write a point in homogeneous coordinates $[v], v=$ $\left(z_{0}, z_{1}, z_{2}\right) \in \mathbb{C}^{3}$. Then $\mathrm{SO}(3)$ acts on $\mathbb{C P}^{2}$ as $[v] \rightarrow[g v]$. One singular orbit is clearly $B_{-}=\mathbb{R P}^{2}=\left\{[v] \mid v \in \mathbb{R}^{3} \subset \mathbb{C}^{3}\right\}$. Let $x_{-}=[(1,0,0)]$ and hence $\mathrm{K}^{-}=\mathrm{S}(\mathrm{O}(1) \mathrm{O}(2))$. One easily checks that $c(t)=[(\cos (t), i \sin (t), 0)]$ is a geodesic in $\mathbb{C P}^{2}$ orthogonal to $B_{-}$at $x_{-}$and that $\mathrm{H}=\mathrm{G}_{c(t)}=\mathbb{Z}_{2}=\langle\operatorname{diag}(-1,-1,1)\rangle$ as long as $0<t<\pi / 4$. When $t=\pi / 4$ we set $x_{+}=[(1 / \sqrt{2}, i / \sqrt{2}, 0)]$ and one shows that $\mathrm{K}^{+}=\mathrm{G}_{x_{+}}=\mathrm{SO}(2)$, which is embedded in $\mathrm{SO}(3)$ as a rotation in the first two coordinates. $B_{+}=\mathrm{G} / \mathrm{K}^{+}$can also be described as the quadric $\sum z_{i}^{2}=0$ in $\mathbb{C P}^{2}$ since $x_{+}$lies in it and the quadric is clearly preserved by the $\mathrm{SO}(3)$ action. After lifting these groups into $\mathrm{S}^{3}$ (and renumbering the coordinates) the group picture for $\mathbb{C P}^{2}$ becomes:

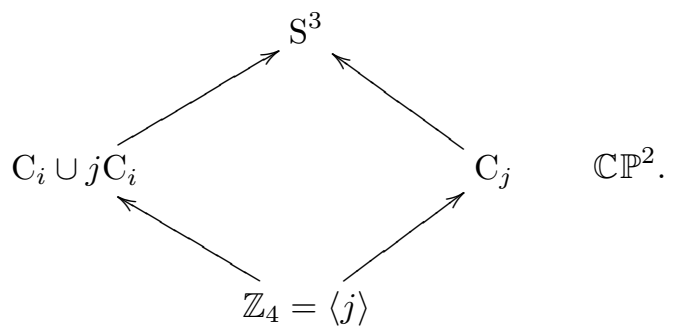


If we compare these group diagrams, one immediately sees that $\mathbb{C P}^{2}$ is an equivariant two fold branched cover of $\mathbb{S}^{4}$, with branching locus (and metric singularity) along the real points $\mathbb{R} \mathbb{P}^{2}$ in $\mathbb{C P}^{2}$ and the Veronese embedding of $\mathbb{R} \mathbb{P}^{2}$ in $\mathbb{S}^{4}$, the covering given by coverings along the orbits of $\mathrm{S}^{3}$.

Next we consider the cohomogeneity one manifold $M_{n}$ defined by the group diagram:

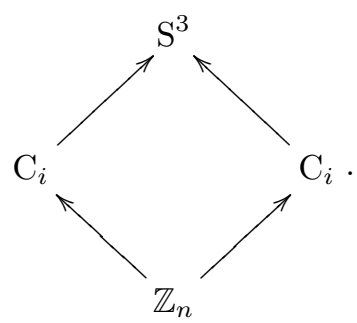

One easily shows that for $n=1$ this is the action on $\mathbb{C} P^{2} \#-\mathbb{C P}^{2}$ obtained as follows: $\mathrm{SU}(2)$ acts on $\mathbb{C P}^{2}$ fixing a point. Take two copies of $\mathbb{C P}^{2}$ with such an action and remove a small ball around the fixed points. If we identify the boundaries with an equivariant diffeomorphism, we obtain the desired action on $\mathbb{C} P^{2} \#-\mathbb{C P}^{2}$. For $n=2$, one easily shows that the group diagram is the one induced by the linear action of $\mathrm{SO}(3)$ on $\mathbb{S}^{2} \times \mathbb{S}^{2}$ given by $A \cdot(v, w)=(A v, A w)$. In both cases we have used the fact (see Lemma 1.3) that any two circles in $S^{3}$ can be conjugated to each other with an element in $\mathrm{N}(\mathrm{H})_{0}=S^{3}$. In general one shows ( $\left.\mathrm{Pa}\right)$ that $M_{n}$ is diffeomorphic to $\mathbb{C P}^{2} \#-\mathbb{C P}^{2}$ for $n$ odd, and to $\mathbb{S}^{2} \times \mathbb{S}^{2}$ for $n$ even, although they are of course not equivariantly diffeomorphic. All actions in (2.3) admit extensions to $\mathrm{S}^{3} \times \mathrm{S}^{1}$ given by the group diagrams $\mathrm{S}^{1}=\left\{\left(e^{i n \theta}, e^{i \theta}\right)\right\} \subset\left\{\mathrm{T}^{2}, \mathrm{~T}^{2}\right\} \subset \mathrm{S}^{3} \times \mathrm{S}^{1}$.

The only further action where both orbits have cohomogeneity two is the product of a transitive action and a cohomogeneity one action on $\mathbb{S}^{2} \times \mathbb{S}^{2}$; i.e., it is given by

$$
\mathrm{SO}(2) \times\{1\} \subset\{\mathrm{SO}(2) \mathrm{SO}(2), \mathrm{SO}(2) \mathrm{SO}(2)\} \subset \mathrm{SO}(3) \mathrm{SO}(2) .
$$

We now proceed to quickly record the remaining cohomogeneity one actions on 1-connected 4-manifolds.

The first one is the suspension action on $\mathbb{S}^{4} \subset \mathbb{R}^{5}=\mathbb{R}^{4} \oplus \mathbb{R}$ with diagram

$$
\{1\} \subset\left\{S^{3}, S^{3}\right\} \subset S^{3}
$$

and its extensions

$$
\mathrm{U}(1) \subset\{\mathrm{U}(2), \mathrm{U}(2)\} \subset \mathrm{U}(2) \text { and } \mathrm{SO}(3) \subset\{\mathrm{SO}(4), \mathrm{SO}(4)\} \subset \mathrm{SO}(4) .
$$

The second is a sum action on $\mathbb{S}^{4} \subset \mathbb{R}^{5}=\mathbb{R}^{3} \oplus \mathbb{R}^{2}$ with diagram

$$
\{1\} \times \mathrm{SO}(2) \subset\{\{1\} \times \mathrm{SO}(3), \mathrm{SO}(2) \times \mathrm{SO}(2)\} \subset \mathrm{SO}(2) \mathrm{SO}(3) .
$$

The third action is the action on $\mathbb{C P}^{2}$ with a fixed point; i.e., it is induced from the sum action on $\mathbb{S}^{5} \subset \mathbb{C} \oplus \mathbb{C}^{2}$ and has the diagram

$$
\{1\} \subset\{\mathrm{SU}(2), \mathrm{U}(1)\} \subset \mathrm{SU}(2)
$$

and its extension

$$
\mathrm{U}(1) \subset\{\mathrm{U}(2), \mathrm{U}(1) \mathrm{U}(1)\} \subset \mathrm{U}(2) .
$$

According to Parker $\mathrm{Pa}$, this exhausts all cohomogeneity one actions on 1-connected 4-manifolds. But notice that the action of $\mathrm{SO}(3)$ on $\mathbb{C P}^{2}$ and all actions with $\mathrm{G}=\mathrm{U}(2)$ were left out in his classification. For a complete list, see $[\mathrm{Ho}$, where 
a classification of simply connected cohomogeneity one manifolds of dimension at most 7 was carried out.

\section{TOPOLOGICAL CLASSIFICATION}

The purpose of this section is to review the classification of vector bundles over simply connected closed 4-manifolds $M$, and to relate it to our setting. Specifically, the classification is expressed in terms of characteristic classes, and for our purposes it is essential that we can read this information off from the cohomology of the total space of the corresponding principal bundles. Since all vector bundles over $M$ are orientable, this amounts to a classification of principal $\mathrm{SO}(k)$ bundles over such manifolds.

(3.1)

Principle $\mathrm{SO}(2)$ bundles $P$ are classified by their Euler class $e(P) \in H^{2}(M, \mathbb{Z})$

and any such class is realized by a unique principal bundle.

In the case of principal $\mathrm{SO}(3)$ bundles, it is well known that they are classified by their second Stiefel-Whitney class $w_{2}(P) \in H^{2}\left(M, \mathbb{Z}_{2}\right)$ and their first Pontryagin class $p_{1}(P) \in H^{4}(M, \mathbb{Z})=\mathbb{Z}$ (cf. [FU] and [DW]). Fixing an orientation on $M$, we identify $p_{1}$ with the integer $k=p_{1}(P)([M])$. Here $w_{2}$ can be chosen to be arbitrary, but the values of $p_{1}$ are restricted to a congruence class mod 4 . To see which one is allowed for a given value of $w_{2}(P)$, choose a principal $\mathrm{SO}(2)$ bundle $P^{*}$ over $M$ whose Euler class $e \in H^{2}(M, \mathbb{Z})$ reduced mod 2 is equal to $w_{2}$ and let $P$ be the principal $\mathrm{SO}(3)$ bundle that we obtain by extending the structure group of $P^{*}$. Then $w_{2}(P)=w_{2}$ and $p_{1}(P)=e^{2}$ (see [Mi, 15.8]). Thus we have:

Proposition 3.2. A principal $\mathrm{SO}(3)$ bundle $P$ over a simply connected 4-manifold $M$ is determined by its second Stiefel-Whitney class $w_{2}$ and its first Pontryagin class $p_{1}$. Here $w_{2}$ can take any value, and $p_{1}$ can take any value congruent to $e^{2}$ mod 4, where $e$ is the Euler class of a principal circle bundle with Stiefel-Whitney class $w_{2}$.

In particular, for bundles over $\mathbb{C P}^{2}$, we have $p_{1} \equiv 1 \bmod 4$ if $w_{2} \neq 0$ and $p_{1} \equiv$ $0 \bmod 4$ if $w_{2}=0$, as long as we choose the orientation class on $\mathbb{C P}^{2}$ to be the square of a generator in dimension 2 .

In section one we saw how principal $\mathrm{SO}(4)$ bundles $P$ are in one-to-one correspondence with a pair of $\mathrm{SO}(3)$ bundles $P^{ \pm}$with the same $w_{2}$. They are classified according to the following well-known result (cf. [DW]):

Proposition 3.3. A principal $\mathrm{SO}(4)$ bundle $P$ over a simply connected 4-manifold $M$ is determined by $w_{2}(P), p_{1}(P)$, and the Euler class $e(P)$. Here $w_{2}(P)$ can take any value, whereas $p_{1}(P)$ and $e(P)$ are restricted via Proposition 3.2 and (1.10).

In particular for $\mathrm{SO}(4)$ bundles over $\mathbb{C P}^{2}$, the allowed values of these invariants are given by $p_{1}(P)=2 k+2 l, e(P)=k-l$ for $k, l \in \mathbb{Z}$ in the spin case, and by $p_{1}(P)=2 k+2 l+1, e(P)=k-l$ in the nonspin case.

The classification of the remaining principal bundles is provided by the following well-known fact (cf. DW]):

Proposition 3.4. For $k \geq 5$, a principal $\mathrm{SO}(k)$ bundle $P$ over a simply connected 4-manifold $M$ is determined by $w_{2}(P), w_{4}(P)$ and $p_{1}(P)$. 
Recall that a $k$-dimensional vector bundle over $M^{4}$ is the direct sum of a 4 dimensional vector bundle with a trivial one and that for a 4-dimensional vector bundle $w_{4}(P) \equiv e(P) \bmod 2$. This completely determines the allowed values of the invariants in Proposition 3.4

In our later applications we only need to consider $M=\mathbb{S}^{4}$ and $M=\mathbb{C P}^{2}$ and in these cases one obtains the following table for the allowed values:

TABle A

\begin{tabular}{|c|c|c|}
\hline$p_{1}$ & $w_{2}$ & $w_{4}$ \\
\hline $0 \bmod 4$ & 0 & 0 \\
\hline $2 \bmod 4$ & 0 & $\neq 0$ \\
\hline $1 \bmod 4$ & $\neq 0$ & 0 \\
\hline $3 \bmod 4$ & $\neq 0$ & $\neq 0$ \\
\hline
\end{tabular}

In particular, we see that the values of $w_{2}$ and $w_{4}$ happen to be determined by $p_{1}$.

Our strategy in determining which principal $\mathrm{SO}(k)$ bundles $P$ admit a commuting lift of a given cohomogeneity one action is to use their description in Lemma 1.5 and compute the possible corresponding characteristic classes. As indicated earlier, it is crucial for us that these in turn can be expressed in terms of the topology of the total space $P$ according to the following result:

Proposition 3.6. Let $M$ be a compact, simply connected, 4-dimensional manifold with second Betti number $b$, and $P \rightarrow M$ a principal $\mathrm{SO}(k)$ bundle with $s=\left|p_{1}(P)([M])\right|$. Then

(a) $w_{2} \neq 0$ if and only if $P$ is simply connected.

(b) $p_{1}(P) \neq 0$ if and only if $H^{4}(P, \mathbb{Z})$ is finite, and in that case $H^{3}(P, \mathbb{Z})=0$.

(c) If $p_{1}(P) \neq 0$, then $\left|H^{4}(P, \mathbb{Z})\right|=2^{b-1} s$ if $k=3$ and $\left|H^{4}(P, \mathbb{Z})\right|=2^{b} s$ if $k \geq 5$.

Proof. As we observed in the proof of Proposition [1.8, it follows from the long homotopy sequence of the principal bundle that $\pi_{1}(P)=0$ precisely when $w_{2} \neq 0$.

To compute $H^{4}(P)$ we use the spectral sequence for the principal bundle $\mathrm{SO}(k)$ $\rightarrow P \rightarrow M$. Let $\left(E_{r}, d_{r}\right)$ be the spectral sequence of this bundle, and $\left(\tilde{E}_{r}, \tilde{d}_{r}\right)$ the spectral sequence of the universal principal $\mathrm{SO}(k)$ bundle $\mathrm{SO}(k) \rightarrow E \rightarrow B_{\mathrm{SO}(k)}$ with contractible total space $E$. The classifying map $f: M \rightarrow B_{\mathrm{SO}(k)}$ induces maps between these spectral sequences, and we will use the naturality of the differentials.

We first assume that $k \geq 5$ and examine the spectral sequence for the universal bundle. It is well known that for the cohomology groups $H^{*}(\mathrm{SO}(k), \mathbb{Z})$ one has: $H^{1}=0, H^{2}=\mathbb{Z}_{2}, H^{3}=\mathbb{Z}, H^{4}=\mathbb{Z}_{2}$ (see, e.g., [Ha], p. 292). Similarly, the groups $H^{*}\left(B_{\mathrm{SO}(k)}, \mathbb{Z}\right)$ are given by $H^{1}=H^{2}=0, H^{3}=H^{5}=\mathbb{Z}_{2}$. Moreover, $H^{4}\left(B_{\mathrm{SO}(k)}\right)=\mathbb{Z}$ with generator $p_{1}(E)$ (see e.g. [Mi, p.182]). Hence $\tilde{E}_{2}^{2,2}=H^{2}\left(B_{\mathrm{SO}(3)}, H^{2}(\mathrm{SO}(3))\right)=H^{2}\left(B_{\mathrm{SO}(3)}, \mathbb{Z}_{2}\right)=\mathbb{Z}_{2}$. and since $E$ is contractible, $\tilde{d}_{2}: \tilde{E}_{2}^{0,3}=H^{3}(\mathrm{SO}(3), \mathbb{Z})=\mathbb{Z} \rightarrow \tilde{E}_{2}^{2,2}=\mathbb{Z}_{2}$ must be onto and hence $\tilde{d}_{2} x \neq 0$ if $x \in \tilde{E}_{2}^{0,3}$ is a generator. If we reduce the coefficients from $\mathbb{Z}$ to $\mathbb{Z}_{2}$ 
in the spectral sequence, this element $\tilde{d}_{2} x$ is nonzero, and hence corresponds to the second Stiefel-Whitney class. Thus we can write $\tilde{d}_{2} x=w_{2}(E)$. Furthermore, $\tilde{d}_{4}: \tilde{E}_{4}^{0,3}=\mathbb{Z} \rightarrow \tilde{E}_{4}^{4,0}=\mathbb{Z}$ must take the generator $2 x$ in $\tilde{E}_{4}^{0,3}$ to the generator $p_{1}(E)$ in $\tilde{E}_{4}^{4,0}$, i.e. $d_{4}(2 x)=p_{1}(E)$.

Notice that under the map $f, f^{*}\left(p_{1}(E)\right)=p_{1}(P) \in H^{4}(M, \mathbb{Z})$ and $f^{*}\left(w_{2}(E)\right)=$ $w_{2}(P) \in H^{2}\left(M, \mathbb{Z}_{2}\right)$ and hence the naturality of the spectral sequence implies that $d_{2} x=w_{2}(P)$ and $d_{4}(2 x)=p_{1}(P)$. Therefore, if $w_{2}(P) \neq 0$, we have $d_{2} x \neq 0$ and $2 x$ becomes the generator in $E_{3}^{0,3}=\mathbb{Z}$ with $d_{4}(2 x)=p_{1}(P)$. Thus $E_{5}^{4,0}=E_{\infty}^{4,0}=\mathbb{Z}_{s}$ and $E_{5}^{2,2}=E_{\infty}^{2,2}=\mathbb{Z}_{2}^{b-1}, E_{5}^{0,4}=E_{\infty}^{0,4}=\mathbb{Z}_{2}$ and hence $\left|H^{4}(P, \mathbb{Z})\right|=2^{b} s$ if $p_{1}(P) \neq$ 0 . If, on the other hand, $p_{1}(P)=0, E_{\infty}^{4,0}=\mathbb{Z}$ which survives in $H^{4}(P, \mathbb{Z})$.

If $w_{2}(P)=0$, then $d_{2} x=0$ and hence $x$ is a generator in $E_{3}^{0,3}=\mathbb{Z}$ with $d_{4} x=\frac{1}{2} p_{1}(P)$. Thus $E_{\infty}^{4,0}=\mathbb{Z}_{\frac{1}{2} s}$ and $E_{\infty}^{2,2}=\mathbb{Z}_{2}^{b}$ if $p_{1}(P) \neq 0$, and we again obtain $\left|H^{4}(P, \mathbb{Z})\right|=2^{b} k$. As before, if $p_{1}(P)=0, H^{4}(P, \mathbb{Z})$ contains $\mathbb{Z}$.

In both cases, the fact that $H^{3}(P, \mathbb{Z})=0$ if $p_{1}(P) \neq 0$ now immediately follows from the spectral sequence for $P$.

The only change that occurs when $k=3$ is that $E_{\infty}^{0,4}=H^{4}(\mathrm{SO}(k), \mathbb{Z})=0$ and $H^{5}\left(B_{\mathrm{SO}(k)}\right)=0$. Arguing as above finishes the proof.

This leaves undetermined the sign of $p_{1}$. Notice that in the case of $k=3$ and $w_{2}=0$, the two-fold spin cover of $P$ is an $\mathrm{S}^{3}$ bundle over $M$ whose Pontryagin class is four times the Euler class since $H^{4}\left(B_{\mathrm{SO}(3)}\right) \rightarrow H^{4}\left(B_{\mathrm{SU}(2)}\right)$ is multiplication by 4. Hence one can change the sign by changing the orientation of the sphere bundle. Thus, if one admits a lift, so does the other. In the case of $k=3$ and $w_{2} \neq 0$ on the other hand, the sign of $p_{1}(P)$ is determined since $p_{1}(P) \equiv e^{2} \bmod 4$.

For $k>4$ the situation is more complicated. One can show that the two bundles obtained by changing the sign of $p_{1}$ have the same cohomology groups. As was pointed out to us by N. Kitchloo, the homotopy type of the two bundles is different though and can be distinguished by the Steenrod square $S q^{2}$. This issue will arise for us only in the case of the $\mathrm{SU}(2)$ action on $\mathbb{C P}^{2}$ with a fixed point, where we will leave it as an open question. Notice though that this does not affect the geometric applications in the Introduction since for this action only one of the singular orbits has codimension two.

The topology of principal bundles $P$ that admit a commuting lift can, in our case, be analyzed in terms of their description (1.4) as cohomogeneity one manifolds. In particular, their decomposition as a union of two disc bundles over the singular orbits allows the use of known topological tools and representation theory to complete our task in the next two sections.

\section{LifTS YIELDING BUNDLES WITH NONNEGATIVE CURVATURE}

The following result obtained in GZ is the basic source for nonnegative curvature examples we use here.

Theorem 4.1. Any cohomogeneity one manifold with codimension two singular orbits admits a nonnegatively curved invariant metric.

A key property of the principal bundle construction $P \rightarrow M$ for cohomogeneity one manifolds (see Lemma 1.5) is that the normal bundles to the singular orbits in $P$ are the pullbacks of the normal bundles of the singular orbits in the base $M$. In 
particular, $P$ admits an invariant metric of nonnegative curvature if the singular orbits of the base have codimension two.

We will now begin our classification of principal $\mathrm{SO}(k)$ bundles admitting commuting lifts. This section is devoted to lifts of actions where both singular orbits have codimension two.

Recall ([PS], $[\mathrm{HY}]$ ) that any group acting on a simply connected manifold $M$ admits a unique commuting lift to any torus bundle over $M$. We can therefore assume that $k \geq 3$.

We first deal with the simpler case of bundles over $\mathbb{S}^{2} \times \mathbb{S}^{2}$ and $\mathbb{C P}^{2} \#-\mathbb{C P}^{2}$ given by the cohomogeneity one actions (2.3), their extensions to $S^{3} \times S^{1}$, or (2.4). Since for these actions $\mathrm{K}^{-}=\mathrm{K}^{+}$is a maximal torus of $G$, Lemma 1.7 (a) implies that the structure group reduces to a torus. Thus we have:

Theorem 4.2. A principal $\mathrm{SO}(k)$-bundle over $\mathbb{S}^{2} \times \mathbb{S}^{2}$ or $\mathbb{C P}^{2} \#-\mathbb{C P}^{2}$ with any of its cohomogeneity one actions admits a commuting lift if and only if it admits a reduction to a torus.

It is worth noticing that over $\mathbb{S}^{2} \times \mathbb{S}^{2}$ and $\mathbb{C P}^{2} \#-\mathbb{C P}^{2}$ quite a few of the principal $\mathrm{SO}(k)$ bundles already arise in this trivial fashion. More precisely, we have:

Theorem 4.3. $\quad$ (a) A principal $\mathrm{SO}(3)$-bundle over $\mathbb{S}^{2} \times \mathbb{S}^{2}$ admits a reduction to $\mathrm{SO}(2)$ unless $w_{2}=0$ and $p_{1} \equiv 4 \bmod 8(0 \bmod 4$ are the allowed values $)$.

(b) A principal $\mathrm{SO}(3)$-bundle over $\mathbb{C P}^{2} \#-\mathbb{C P}^{2}$ admits a reduction to $\mathrm{SO}(2)$ unless $w_{2}=0$ and $p_{1} \equiv 8 \bmod 16$, or $w_{2}=(1,1)$ and $p_{1} \equiv 4 \bmod 8($ in both cases the allowed values for $p_{1}$ are $\left.0 \bmod 4\right)$.

(c) Every principal $\mathrm{SO}(k)$ bundle over $\mathbb{S}^{2} \times \mathbb{S}^{2}$ and $\mathbb{C P}^{2} \#-\mathbb{C P}^{2}$ with $k \geq 6$ admits a reduction to a 3 -torus.

Proof. If $M=\mathbb{S}^{2} \times \mathbb{S}^{2}$, the $\mathrm{SO}(2)$ principal bundle over $M$ with Euler class $e=$ $(a, b) \in H^{2}(M, \mathbb{Z})=\mathbb{Z} \oplus \mathbb{Z}$ has first Pontryagin class $p_{1}=(a, b)^{2}[M]=2 a b$. We now use Proposition 3.2 to determine the allowed values of $p_{1}$ for an $\mathrm{SO}(3)$ principal bundle with a given Stiefel-Whitney class $w_{2}=(a, b) \bmod 2$. The circle bundle with $e=(2 k, 1)($ resp. $e=(1,2 k))$ has $p_{1}=4 k$ and $w_{2}=(0,1)$ (resp. $\left.(1,0)\right)$ which are precisely the allowed values in this case. Thus all $\mathrm{SO}(3)$ bundles with $w_{2}=(0,1)$ and $(1,0)$ admit a reduction to $\mathrm{SO}(2)$. The same holds in the case of $w_{2}=(1,1)$ using the 2 -plane bundle with $e=(2 k+1,1)$. In all 3 cases, Proposition 1.8 implies that a bundle with $k=4$ admits a reduction to a 2 -torus since both $P^{ \pm}$admit a reduction to $\mathrm{SO}(2)$. For $k \geq 5$ the structure group reduces to $\mathrm{SO}(4)$ and hence to a 2-torus.

If $w_{2}=(0,0)$, then the 2-plane bundle with $e=(2 k, 2 l)$ has $p_{1}=8 k l$. Hence the $\mathrm{SO}(3)$ bundles with $p_{1} \equiv 0 \bmod 8$ reduce to $\mathrm{SO}(2)$ and those with $p_{1} \equiv 4$ $\bmod 8$ do not. If we let $P^{-}$be the $\mathrm{SO}(3)$ bundle with $p_{1}=8 k$ and $P^{+}$the one with $p_{1}=8 l$, then Proposition 3.2 defines an $\mathrm{SO}(4)$ bundle with $w_{2}=(0,0), p_{1}=$ $4 k+4 l$ and $e=2 k-2 l$. Thus, according to Theorem 3.4 any bundle with $k \geq 5$, $w_{2}=(0,0), p_{1} \equiv 0 \bmod 4$ and $w_{4}=0$ admits a reduction to a torus. To see what the precise allowed values are in this case we use Proposition 3.4 Since every $k$ dimensional bundle reduces to $\mathrm{SO}(4)$ and since the $\mathrm{SO}(4)$ bundle with $p_{1}\left(P^{-}\right)=4 k$ and $p_{1}\left(P^{+}\right)=4 l$ has $p_{1}=2 k+2 l$ and $e=k-l$, it follows that for $k \geq 5$ we either have $p_{1} \equiv 0 \bmod 4$ and $w_{4}=0$ or $p_{1} \equiv 2 \bmod 4$ and $w_{4} \neq 0$. In the latter case we can consider the direct sum of 3 two-dimensional bundles with Euler classes 
$e=(1,1), e=(1,0)$ and $e=(2 k, 1)$ which has $w_{2}=(0,0)$ and $p_{1}=4 k+2$ (and thus $\left.w_{4} \neq 0\right)$. Thus its structure group reduces to a 3 -torus.

We indicate the argument for $\mathbb{C P}^{2} \#-\mathbb{C P}^{2}$. The $\mathrm{SO}(2)$ bundle with Euler class $e=(a, b) \in H^{2}(M, \mathbb{Z})=\mathbb{Z} \oplus \mathbb{Z}$ has first Pontryagin class $p_{1}=(a, b)^{2}[M]=a^{2}-b^{2}$. If $w_{2}=(1,0)$ or $(0,1)$ the bundles with $e=(2 k+1,2 k)$, resp. $e=(2 k, 2 k-1)$, have $p_{1}=4 k+1$, resp. $p_{1}=4 k-1$, and these are precisely the allowed values in the case of $k=3$. Thus any $\mathrm{SO}(k)$ bundle with these Stiefel-Whitney classes reduces to a 2 -torus.

If $w_{2}=(1,1)$ the 2-plane bundle with $e=(2 k+1,2 k-1)$ has $p_{1}=8 k$, whereas $p_{1} \equiv 0 \bmod 4$ are the allowed values in the case of $k=3$. This gives rise to 5 -plane bundles with $w_{2}=(1,1), p_{1} \equiv 0 \bmod 4$ and $w_{4}=0$ whose structure group reduces to a 2 -torus. To produce the remaining bundles with $p_{1} \equiv 2 \bmod 4$ and $w_{4} \neq 0$ we consider the direct sum of 3 two-dimensional bundles with Euler classes $e=(1,1)$, $e=(1,0)$ and $e=(2 k+1,2 k)$.

In the case $w_{2}=(0,0)$ the 2-plane bundle with $e=(2 k, 2 l)$ has $p_{1}=4(k+l)(k-l)$ and $0 \bmod 4$ are the allowed values. One easily sees that this can take on any value of $0,4,12 \bmod 16$, but no value of $8 \bmod 16$. This gives rise, via Proposition 3.2 . to $\mathrm{SO}(5)$ bundles with $p_{1} \equiv 0 \bmod 4$ and hence $w_{4}=0$ whose structure group reduces to a torus. On the other hand the 5 -dimensional bundle which is the direct sum of the two-dimensional bundles with $e=(2 k+1,2 k)$ and $e=(1,0)$ and a trivial bundle has $p_{1}=4 k+2$ and hence $w_{4} \neq 0$. Thus in this case any $\mathrm{SO}(k)$ bundle with $k \geq 5$ reduces to a 2 -torus.

Combining the above 3 theorems, we obtain:

Corollary 4.4. Every vector bundle over $\mathbb{S}^{2} \times \mathbb{S}^{2}$ and $\mathbb{C P ^ { 2 }} \#-\mathbb{C P}^{2}$ with fiber dimension at least 6 has a complete metric with nonnegative curvature.

We finally observe that the examples of nonnegative curvature in the above results can be easily obtained by direct methods, avoiding Theorem 4.1 and Theorem 4.2. Since this argument also generalizes to the remaining nonnegatively curved 4-manifold $\mathbb{C} \mathbb{P}^{2} \# \mathbb{C} \mathbb{P}^{2}$, which does not admit a cohomogeneity one action, we present it here.

Theorem 4.5. Any principal $\mathrm{SO}(k)$ bundle over $\mathbb{S}^{2} \times \mathbb{S}^{2}$ or $\mathbb{C P}^{2} \# \pm \mathbb{C P}^{2}$ whose structure group reduces to a torus admits an $\mathrm{SO}(k)$ invariant metric with nonnegative curvature.

Proof. As was shown in $[\mathrm{Ta}$, these three manifolds can be written as a quotient of $\mathrm{S}^{3} \times \mathrm{S}^{3}$ by an action of a 2 -torus. In the case of $\mathbb{S}^{2} \times \mathbb{S}^{2}$, this is of course simply the right action by a maximal torus. The manifold $\mathbb{C P}^{2} \#-\mathbb{C P}^{2}$ is the quotient under the action $(z, w) \star\left(q_{1}, q_{2}\right)=\left(z q_{1}, z q_{2} w\right)$, where $\left(q_{1}, q_{2}\right) \in \mathrm{S}^{3} \times \mathrm{S}^{3}$ and $(z, w) \in \mathrm{T}^{2}$. For $\mathbb{C P}^{2} \# \mathbb{C} \mathbb{P}^{2}$ the action $(z, w) \star\left(q_{1}, q_{2}\right)=\left(z w q_{1} w^{2}, \bar{z} w q_{2} \bar{z}^{2}\right)$ suffices. Thus in all 3 cases, $\mathrm{S}^{3} \times \mathrm{S}^{3}$ is the total space of a principal 2-torus bundle over $M$ and a bi-invariant metric is $\mathrm{T}^{2}$-invariant. Since $H^{2}(M, \mathbb{Z}) \simeq \mathbb{Z}^{2}$, this is the only 2torus bundle with simply connected total space and any other 2-torus bundle is a quotient of $\mathrm{S}^{3} \times \mathrm{S}^{3}$ by a finite subgroup of $\mathrm{T}^{2}$. A $\mathrm{T}^{\ell}$-bundle with $\ell>2$ is simply a product of a 2 -torus bundle with $\mathrm{T}^{\ell-2}$. For a circle bundle, one observes that circle bundles with simply connected total space can be described as $\mathrm{S}^{3} \times \mathrm{S}^{3} / \mathrm{S}_{p, q}^{1}$, where $\mathrm{S}_{p, q}^{1} \subset \mathrm{T}^{2}$ is a circle of slope $(p, q)$ with $\operatorname{gcd}(p, q)=1$. Indeed, in terms of appropriate generators $x, y$ of $H^{2}(M, \mathbb{Z})$ given by transgression of the natural 
generators in $H^{1}\left(\mathrm{~T}^{2}, \mathbb{Z}\right)$, the Euler class of such a bundle is $-q x+p y$ and the total space of a circle bundle over a simply connected base is simply connected if and only if its Euler class is primitive. All other circle bundles are given by a quotient of this bundle by a finite subgroup of $\mathrm{S}^{1}$.

Thus in all cases, a $\mathrm{T}^{\ell}$ principal bundle $P$ admits an invariant metric with nonnegative curvature. This implies that the extension $P \times_{\mathrm{T}^{\ell}} \mathrm{SO}(k)$ admits an $\mathrm{SO}(k)$-invariant metric with nonnegative curvature.

Remarks. (a) The proof can be applied to bundles over $\mathbb{C P}^{n} \# \pm \mathbb{C P}^{n}$ as well. In particular any 2-plane bundle over these manifolds admits nonnegative curvature. In the case of $\mathbb{C} \mathbb{P}^{n} \#-\mathbb{C P}^{n}$ this was first shown in Ya.

(b) One can carry out an analysis as in Theorem 4.3 for $\mathbb{C P}^{2} \# \mathbb{C P}^{2}$ as well. But in this case, a circle bundle with $e=(a, b)$ has first Pontryagin class $p_{1}=a^{2}+b^{2}$, and hence only bundles with nonnegative Pontryagin class are obtained. It follows that every $\mathrm{SO}(k)$ bundle with $k \geq 10$ and $p_{1} \geq 0$ admits a reduction to a torus and hence a metric with nonnegative curvature.

We now proceed to consider the most interesting case, that of $\mathrm{SO}(3)$ principal bundles over $\mathbb{C P}^{2}$, since it has the geometric applications in Theorems $\mathrm{A}$ and $\mathrm{B}$ in the Introduction. The only case where both singular orbits have codimension two is the action in (2.2). Since we know that it admits a lift when the structure group reduces to $\mathrm{SO}(2)$, we may assume that the image of $\mathrm{K}^{ \pm}$in $\mathrm{SO}(3)$ is not contained in an $\mathrm{SO}(2)$. It is then not hard to see that the only possible group diagram as in (1.4) defining an $\mathrm{SO}(3)$ principal bundle over $\mathbb{C P}^{2}$ is given by:

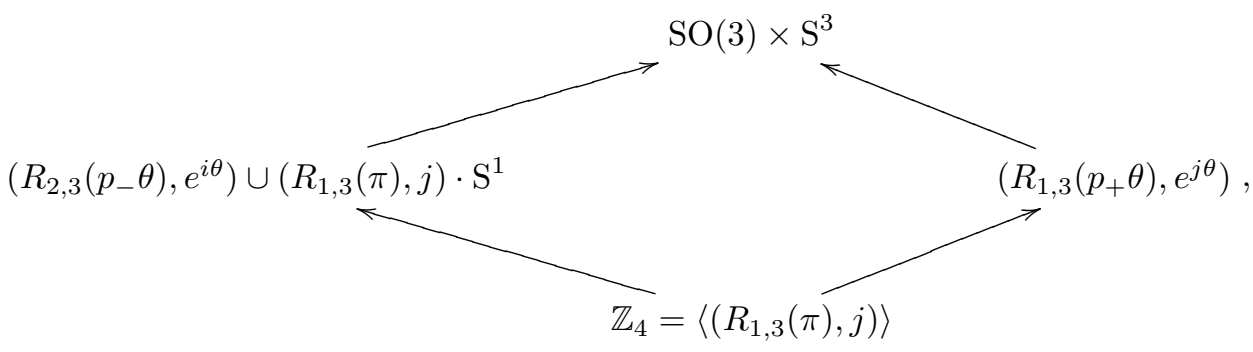

where $p_{-}$is even and $p_{+} \equiv 2 \bmod 4$ in order for the diagram to be consistent. Here and in what follows $\mathrm{S}^{1}$ will denote the identity component of the group $\mathrm{K}^{-}$, and we will use $R_{j, k}(\theta)$ to denote the group of rotations by an angle $\theta$ in the 2-plane spanned by $j, k$.

To characterize these bundles topologically we show:

Theorem 4.7. The principal $\mathrm{SO}(3)$ bundle over $\mathbb{C P}^{2}$ defined by (4.6) has Pontryagin class $p_{1}=\frac{1}{4}\left(p_{+}^{2}-p_{-}^{2}\right)$ and is spin if and only if $p_{ \pm} \equiv 2 \bmod 4$. Furthermore, in the spin case, these are precisely the principal bundles which are obtained as pullback of $\mathrm{SO}(3)$ principal bundles over $\mathbb{S}^{4}$ under the two-fold branched cover $\mathbb{C P}^{2} \rightarrow \mathbb{S}^{4}$.

Proof. Let us first consider the case where $p_{-} \equiv 2 \bmod 4$ and $p_{+} \equiv 2 \bmod 4$. To see that the manifold is spin, consider the $\mathrm{S}^{3}$ principal bundle over $\mathbb{C P}^{2}$ whose total 
space is the cohomogeneity one manifold with the following group picture:

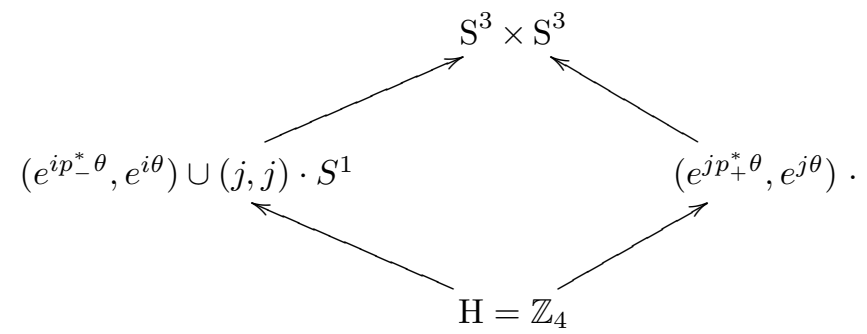

In order for this group diagram to be consistent, we need $p_{-}^{*}$ and $p_{+}^{*}$ odd and $\mathrm{H}=$ $\langle(j, j)\rangle$ if $p_{+}^{*} \equiv 1 \bmod 4$ and $\mathrm{H}=\langle(-j, j)\rangle$ if $p_{+}^{*} \equiv 3 \bmod 4$. If we set $p_{ \pm}^{*}=p_{ \pm} / 2$, the group diagram (4.8) is a lift of (4.6) and hence the manifold is spin.

Now define a two-fold branched cover of (4.8) onto the cohomogeneity one manifold defined by

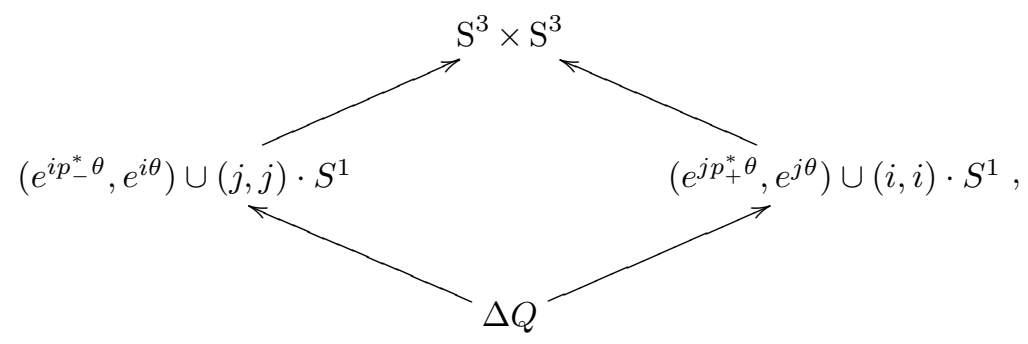

where we need $p_{ \pm}^{*}$ to be odd for the group diagram to be consistent, as long as we allow a sign change in some of the components of $\Delta Q$. Notice that (4.9) is an $\mathrm{S}^{3}$ principal bundle over $\mathbb{S}^{4}$ since after dividing by $S^{3} \times 1$ we obtain the cohomogeneity one picture (2.1) for $\mathbb{S}^{4}$. This two-fold branched cover from (4.8) to (4.9) becomes the two-fold branched cover $\mathbb{C P}^{2} \rightarrow \mathbb{S}^{4}$ after dividing by $\mathrm{S}^{3} \times 1$. In GZ] it was shown that every $\mathrm{S}^{3}$ principal bundle over $\mathbb{S}^{4}$ is of the form (4.9) and has Euler class $e=\frac{1}{8}\left(\left(p_{+}^{*}\right)^{2}-\left(p_{-}^{*}\right)^{2}\right)$ and hence Pontryagin class $p_{1}=\frac{1}{2}\left(\left(p_{+}^{*}\right)^{2}-\left(p_{-}^{*}\right)^{2}\right)$. Since furthermore $\mathbb{C P}^{2} \rightarrow \mathbb{S}^{4}$ induces multiplication by two in dimension 4 , it follows that $p_{1}=\frac{1}{4}\left(p_{+}^{2}-p_{-}^{2}\right)$.

We now consider the manifolds $P$ described by (4.6) where $p_{-} \equiv 0 \bmod 4$ and $p_{+} \equiv 2 \bmod 4$. We claim that these are $\mathrm{SO}(3)$ principal bundles over $\mathbb{C P}^{2}$ which are not spin. By Proposition 3.6 this amounts to showing that $P$ is simply connected. We can determine $\pi_{1}(P)$ by applying van Kampen to the decomposition $P=P_{-} \cup$ $P_{+}, P_{-} \cap P_{+}=P_{0}$ provided by its cohomogeneity one description. Clearly $P_{ \pm}$ is homotopy equivalent to $\mathrm{L} \times \mathrm{G} / \mathrm{K}^{ \pm}$and $P_{0}$ to $\mathrm{L} \times \mathrm{G} / \mathrm{H}$, where $\mathrm{L}=\mathrm{SO}(3)$ and $\mathrm{G}=\mathrm{S}^{3}$ according to our recipe. We now need to compute $\pi_{1}\left(\mathrm{~L} \times \mathrm{G} / \mathrm{K}^{ \pm}\right)$and their inclusion into $\pi_{1}(\mathrm{~L} \times \mathrm{G} / \mathrm{H})$. This is easiest done if we write the orbits as quotients of $S^{3} \times S^{3}$ since then the fundamental group is isomorphic to the group of components of the isotropy group. Now observe that for the preimage of $\mathrm{K}^{+}$into $\mathrm{S}^{3} \times \mathrm{S}^{3}$, the component group can be represented by $(-1,1)$ since $p_{+} \equiv 2 \bmod 4$. For the group $\mathrm{K}^{-}, p_{-} \equiv 0 \bmod 4$ implies that $(-1,1) \in \mathrm{K}_{0}^{-}$and hence $\pm(j, j)$ and $\pm(1,1)$ represent the component group. Finally, for $\mathrm{H}$, its preimage is generated by $(j, j)$ and $(-1,1)$. Altogether, we conclude that $P$ is simply connected and thus $w_{2} \neq 0$. 
To compute the integer $p_{1}(P)$ we use Proposition 3.6. Since $w_{2} \neq 0$, Lemma 3.2 implies that $p_{1}(P) \equiv 1 \bmod 4$. In particular, $p_{1}(P) \neq 0$, and by Proposition 3.6. $H^{4}(P, \mathbb{Z})$ is finite with $\left|p_{1}(P)\right|=\left|H^{4}(P, \mathbb{Z})\right|$. In order to compute $\left|H^{4}(P, \mathbb{Z})\right|$, we apply the Mayer-Vietoris sequence to the decomposition $P=P_{-} \cup P_{+}$. In particular, we need the cohomology groups of the pieces:

Lemma 4.10. The cohomology groups of $P_{-}, P_{+}$and $P_{0}$ satisfy:

(a) $P_{-}$has $H^{3}\left(P_{-}\right)=\mathbb{Z} \oplus \mathbb{Z}_{2}$ and $H^{4}\left(P_{-}\right)=0$.

(b) $P_{+} \simeq \mathbb{S}^{2} \times \mathrm{SO}(3)$ and hence $H^{3}\left(P_{+}\right)=\mathbb{Z}$ and $H^{4}\left(P_{+}\right)=\mathbb{Z}_{2}$.

(c) $P_{0} \simeq\left(\mathbb{S}^{3} / \mathbb{Z}_{4}\right) \times \mathrm{SO}(3)$ and hence $H^{3}\left(P_{0}\right)=\mathbb{Z} \oplus \mathbb{Z} \oplus \mathbb{Z}_{2}$ and $H^{4}\left(P_{0}\right)=\mathbb{Z}_{2}$.

Proof. Let $B_{ \pm}=\mathrm{G} / \mathrm{K}^{ \pm} \subset \mathbb{C P}^{2}$. First note that the restriction maps $H^{2}\left(\mathbb{C P}^{2}, \mathbb{Z}_{2}\right)$ $\rightarrow H^{2}\left(B_{ \pm}, \mathbb{Z}_{2}\right)$ are 0 in the case of $B_{+}=\mathbb{S}^{2}$, and an isomorphism in the case of $B_{-}=$ $\mathbb{R} \mathbb{P}^{2}$. Indeed, this follows from the Mayer-Vietoris sequence for the decomposition $M=M_{-} \cup M_{+}$:

$$
0=H^{1}\left(M_{0}\right) \rightarrow H^{2}(M) \rightarrow H^{2}\left(M_{-}\right) \oplus H^{2}\left(M_{+}\right) \rightarrow H^{2}\left(M_{0}\right) \rightarrow H^{3}(M)=0 .
$$

By considering this sequence first over the integers and using $H^{2}\left(M_{-}\right)$ $=H^{2}\left(\mathbb{R P}^{2}\right)=0, H^{2}\left(M_{0}\right)=H^{2}\left(\mathrm{~S}^{3} / \mathbb{Z}_{4}\right)=\mathbb{Z}_{4}$, it follows that $H^{2}(M, \mathbb{Z}) \rightarrow H^{2}\left(M_{+}, \mathbb{Z}\right)$ is multiplication by 4 , and hence mod 2 becomes the 0 map. We then consider this sequence with $\mathbb{Z}_{2}$ coefficients to conclude that $H^{2}\left(M, \mathbb{Z}_{2}\right) \rightarrow H^{2}\left(M_{-}, \mathbb{Z}_{2}\right)$ is an isomorphism.

We next consider $P_{ \pm}$as an $\mathrm{SO}(3)$ bundle over $M_{ \pm}$which is homotopy equivalent to the restriction of this bundle to $B_{ \pm}$. But a principal $\mathrm{SO}(3)$ bundle over a 2complex is classified by $w_{2}$. Hence our previous remark implies that $P_{+} \rightarrow B_{+}$and $P_{0} \rightarrow M_{0}$ are trivial bundles and thus $P_{+} \simeq \mathbb{S}^{2} \times \mathrm{SO}(3)$ and $P_{0} \simeq \mathrm{S}^{3} / \mathbb{Z}_{4} \times \mathrm{SO}(3)$. This determines their cohomology groups.

For $P_{-} \rightarrow B_{-}=\mathbb{R} \mathbb{P}^{2}$, it follows that $w_{2}=1$, and hence it is the unique nontrivial principal $\mathrm{SO}(3)$ bundle over $\mathbb{R P}^{2}$. We can therefore use the following more convenient description for $P_{-}$: Consider $\mathbb{S}^{2} \times \mathrm{S}^{3} / \mathbb{Z}_{4}$, where $\mathbb{Z}_{4}$ acts via the antipodal map on $\mathbb{S}^{2}$ and via left multiplication of $\mathbb{Z}_{4}$ on the unit quaternions $\mathrm{S}^{3}$. This can also be described as $\mathbb{S}^{2} \times \mathrm{SO}(3) / \mathbb{Z}_{2}$, which via the projection on the first factor becomes a principal $\mathrm{SO}(3)$ bundle over $\mathbb{R P}^{2}$. Since the total space has fundamental group $\mathbb{Z}_{4}$, it must be the nontrivial principal bundle. Moreover, it can be viewed as a (nonorientable) $\mathbb{S}^{2}$ bundle over the lens space $S^{3} / \mathbb{Z}_{4}$, via projection onto the second factor. The Euler class of that bundle is easily seen to be $0 \bmod 2$, and hence one computes, by using the Gysin sequence, the cohomology of $\mathbb{S}^{2} \times \mathrm{S}^{3} / \mathbb{Z}_{4}$ with $\mathbb{Z}_{2}$ coefficients to be $\mathbb{Z}_{2}$ in dimensions $0,1,4$ and 5 and $\mathbb{Z}_{2} \oplus \mathbb{Z}_{2}$ in dimensions 2 and 3 . This only leaves the following possibilities for the cohomology with integer coefficients: $H^{3}\left(P_{-}\right)=\mathbb{Z} \oplus \mathbb{Z}_{2}$ and $H^{4}\left(P_{-}\right)=0$.

We now incorporate this information into the Mayer-Vietoris sequence. Denote by $\pi_{ \pm}: P_{0} \rightarrow P_{ \pm}$the projections of the sphere bundle $P_{0} \cong \mathrm{L} \times \mathrm{G} / \mathrm{H} \rightarrow$ $\mathrm{L} \times \mathrm{G} / \mathrm{K}^{ \pm} \cong P_{ \pm}$. We then have:

$$
\begin{aligned}
0=H^{3}(P) & \rightarrow H^{3}\left(P_{-}\right) \oplus H^{3}\left(P_{+}\right)=\mathbb{Z} \oplus \mathbb{Z}_{2} \oplus \mathbb{Z}^{\pi_{-}^{*}-\pi_{+}^{*}} H^{3}\left(P_{0}\right)=\mathbb{Z} \oplus \mathbb{Z} \oplus \mathbb{Z}_{2} \\
& \rightarrow H^{4}(P) \rightarrow \rightarrow H^{4}\left(P_{-}\right) \oplus H^{4}\left(P_{+}\right)=\mathbb{Z}_{2} \rightarrow H^{4}\left(P_{0}\right)=\mathbb{Z}_{2} \rightarrow \cdots
\end{aligned}
$$

where we have used the fact from Proposition 3.6 that $H^{3}(P)=0$. Using the Gysin sequence of the circle bundle $P_{0} \rightarrow P_{+}$, this also implies that $H^{4}\left(P_{+}\right) \rightarrow H^{4}\left(P_{0}\right)$ 
is an isomorphism. It follows that $H^{4}(P)$ is a finite group whose order is equal to the order of the cokernel of the map $\pi_{-}^{*}-\pi_{+}^{*}$ restricted to $\mathbb{Z}^{2}$.

To compute the order of this cokernel, we proceed as in [GZ], diagrams (3.5) and (3.6), and use the same notation. The projection $\eta: \mathrm{L} \times \mathrm{G} \rightarrow \mathrm{L} \times \mathrm{G} / \mathrm{H}=$ $\left(\mathbb{S}^{3} / \mathbb{Z}_{4}\right) \times \mathrm{SO}(3)$ is, as in that case, an 8 -fold cover and induces a map with determinant 8 on $\mathbb{Z} \oplus \mathbb{Z}$. But the projection $\mu_{+}: \mathrm{L} \times \mathrm{G} / \mathrm{K}_{0}^{+}=\mathbb{S}^{2} \times \mathrm{S}^{3} \rightarrow \mathrm{L} \times \mathrm{G} / \mathrm{K}^{+}=$ $\mathbb{S}^{2} \times \mathrm{SO}(3)$ is now a two-fold cover which induces multiplication by 2 on $H^{3}=\mathbb{Z}$. For the projection $\mu_{-}: \mathrm{L} \times \mathrm{G} / \mathrm{K}_{0}^{-} \rightarrow \mathrm{L} \times \mathrm{G} / \mathrm{K}^{-}$, which is now a 4 -fold cover, we can use the description obtained in the proof of Lemma 4.10, which implies that this cover can be described as $\mathbb{S}^{2} \times \mathrm{S}^{3} \rightarrow \mathbb{S}^{2} \times \mathrm{S}^{3} / \mathbb{Z}_{4}$, which induces multiplication by 4 on $H^{3}=\mathbb{Z}$. Finally, observe that the preimage of the circle $\left(R_{1,3}\left(p_{+} \theta\right), e^{j \theta}\right) \subset \mathrm{SO}(3) \times \mathrm{S}^{3}$ is $\left(e^{j \frac{p_{+}}{2} \theta}, e^{j \theta}\right) \subset \mathrm{S}^{3} \times \mathrm{S}^{3}$ since $p_{+}$is even, and similarly for $\mathrm{L} \times \mathrm{G} / \mathrm{K}^{-}$.

Thus we get

$$
\left|p_{1}\right|=\left|H^{4}(P)\right|=\left|\operatorname{cokernel}\left(\pi_{-}^{*}-\pi_{+}^{*}\right)\right|=\frac{1}{8} \operatorname{det}\left(\begin{array}{cc}
-4 & 2 \\
p_{-}^{2} & -\frac{1}{2} p_{+}^{2}
\end{array}\right)=\frac{1}{4}\left|p_{+}^{2}-p_{-}^{2}\right| .
$$

Since $p_{1}(P) \equiv 1 \bmod 4, p_{-} \equiv 0 \bmod 4$ and $p_{+} \equiv 2 \bmod 4$ it follows that $p_{1}=$ $\frac{1}{4}\left(p_{+}^{2}-p_{-}^{2}\right)$. This completes the proof of Theorem 4.7

We can now determine which bundles admit a lift of the action. We leave out the case where the structure group reduces to a torus, since we already know that it always admits a lift in that case.

Corollary 4.11. Let $P \rightarrow \mathbb{C P}^{2}$ be a principal $\mathrm{SO}(k)$ bundle whose structure group does not reduce to a torus and consider the cohomogeneity one action of $\mathrm{SO}(3)$ on $\mathbb{C P}^{2}$.

(a) For $k=3$, the action has a lift unless $w_{2}=0$ and $p_{1} \equiv 4 \bmod 8$.

(b) For $k=4$, the action has a lift unless $w_{2}=0$ and $p_{1}(P)=2 k+2 l, e(P)=$ $k-l$ and $k, l$ not both even.

(c) For $k \geq 5$, the action admits a lift to every principal bundle.

Proof. In the case of $k=3$ and $w_{2} \neq 0$ we need to show, due to Lemma 3.2, that all values of $p_{1}(P) \equiv 1 \bmod 4$ are assumed. But one easily sees that if $p_{-}=4 s$ and $p_{+}=4 r+2$, then $p_{1}=4\left(r^{2}-s^{2}+r\right)+1$ achieves all values of $1 \bmod 4$. Similarly, if $w_{2}=0$, all values of $0 \bmod 8$ are assumed. In the case of $k=4$ the result follows from Theorem 4.7 by applying Lemma 1.6(c), Proposition 1.8, Proposition 3.3 and (1.10).

For $k \geq 5$ we use the general fact that a $k$-dimensional vector bundle over $M^{4}$ is the direct sum of a 4-dimensional vector bundle with a trivial one; i.e., the $\mathrm{SO}(k)$ principal bundle can be viewed as an extension of an $\mathrm{SO}(4)$ bundle. Thus, in the case of $w_{2} \neq 0$, Lemma 1.6 (a) implies that every $\mathrm{SO}(k)$ principal bundle admits a lift.

If $w_{2}=0$ we need to show, due to Proposition 3.4 that $p_{1}$ can achieve every even value. From the case of $k=4$, it follows that every value of $0 \bmod 4$ is assumed. We can now take the direct sum of a 3 -dimensional vector bundle with $w_{2} \neq 0$ and $p_{1}=4 s+1$ with a two-dimensional vector bundle with Euler class one and hence $w_{2} \neq 0$ and $p_{1}=1$, which admits a lift by Lemma 1.6 (c) since both do. By the product formula for Pontryagin classes and Stiefel-Whitney classes, we have $w_{2}=0$ 
and $p_{1}=4 s+2$. Thus every even value of $p_{1}$ is already assumed for 5 -dimensional vector bundles. As we saw in Table A, the value of $w_{4}$ is determined by whether $p_{1}$ is 2 or $0 \bmod 4$.

Theorem 4.7 and Corollary 4.11 imply Theorem $\mathrm{C}$ as well as Theorem $\mathrm{A}$ in the Introduction. For complex vector bundles we can do better since in that case $P^{+}$ reduces to $\mathrm{SO}(2)$ which always has a commuting lift. In the case of $w_{2}=0$, the bundles with $p_{1}\left(P^{-}\right) \equiv 0 \bmod 8$ have a lift and Theorem B follows since by (1.11), $p_{1}\left(P^{-}\right)=c_{1}^{2}-4 c_{2}$ and furthermore one has the general fact that $w_{2}=c_{1} \bmod 2$.

\section{LifTS OF SUM ACTIONS}

In this section we will determine which bundles admit commuting lifts of the cohomogeneity one actions on 1-connected 4-manifolds where at most one singular orbit is of codimension two. Up to extensions there is one such action on $\mathbb{C P}^{2}$ and two on $\mathbb{S}^{4}$.

We begin with the sum action of $\mathrm{SO}(2) \mathrm{SO}(3)$ on $\mathbb{S}^{4}$, where the satisfactory answer is:

Theorem 5.1. The sum action of $\mathrm{SO}(2) \mathrm{SO}(3)$ on $\mathbb{S}^{4}$ admits a commuting lift to every principal $\mathrm{SO}(k)$ bundle.

Proof. As we saw earlier, it suffices to prove the claim for principal $\mathrm{S}^{3}$ bundles. Consider the Brieskorn variety $M_{d}^{7}$ defined by the equations

$$
z_{0}^{d}+z_{1}^{2}+\cdots+z_{4}^{2}=0, \quad\left|z_{0}\right|^{2}+\cdots+\left|z_{4}\right|^{2}=1
$$

It carries a cohomogeneity one action by $\mathrm{SO}(2) \mathrm{SO}(4)$ defined by (cf. [HHs])

$$
\left(e^{i \theta}, A\right)\left(z_{0}, \ldots, z_{4}\right)=\left(e^{2 i \theta} z_{0}, e^{i d \theta} A\left(z_{1}, \ldots, z_{4}\right)^{t}\right) .
$$

The isotropy groups are given by (cf. $[\mathrm{BH}])$ :

$$
\mathrm{K}^{-}=\mathrm{SO}(2) \mathrm{SO}(2), \mathrm{K}^{+}=\mathrm{O}(3) \text { and } \mathrm{H}=\mathbb{Z}_{2} \times \mathrm{SO}(2) .
$$

The normal subgroup $\mathrm{SU}(2) \subset \mathrm{SO}(4)$ acts freely on $\mathbb{R}^{4}$ as left multiplication by quaternions, hence freely on $\mathbb{C}^{4}$ and thus on $M_{d}^{7}$ as well. The quotient is a 4manifold with an induced cohomogeneity one action by $\mathrm{SO}(2) \mathrm{SO}(3)$ with $\mathrm{K}^{-}=$ $\mathrm{SO}(2) \mathrm{SO}(2), \mathrm{K}^{+}=\mathrm{SO}(3)$ (effectively) and $\mathrm{H}=\mathrm{SO}(2)$ and hence must be the cohomogeneity one action (2.6) on $\mathbb{S}^{4}$. Thus $M_{d}^{7}$ is a principal $\mathrm{SU}(2)$ bundle over $\mathbb{S}^{4}$ for which the sum action has a lift. To see which bundle it is, recall that such bundles are classified by their Euler class. Furthermore, it follows from the Gysin sequence that the Euler class (evaluated on a fundamental class) is the order of the fourth cohomology group of the total space. For $M_{d}$ we have (cf. $[\mathrm{Br}, \mathrm{p} .275]$ ) $H^{4}\left(M_{d}, \mathbb{Z}\right)=\mathbb{Z}_{d}$ and hence it is the bundle with Euler class $d$. Thus the action (2.6) on $\mathbb{S}^{4}$ lifts to every principal $\mathrm{SU}(2)$ bundle over $\mathbb{S}^{4}$.

The remaining actions both have fixed points. Here we start with the cohomogeneity one action on $\mathbb{C P}^{2}$ given by the standard $\mathrm{SU}(2)$ and $\mathrm{U}(2)$ action on $\mathbb{C P}^{2}$ with a fixed point; see (2.7). By Lemma 1.7 (b) it suffices to consider the SU(2) action. 
A lift of the cohomogeneity one diagram (2.7) on $\mathbb{C P}^{2}$ to a principal $\mathrm{SO}(k)$ bundle must be given by a diagram

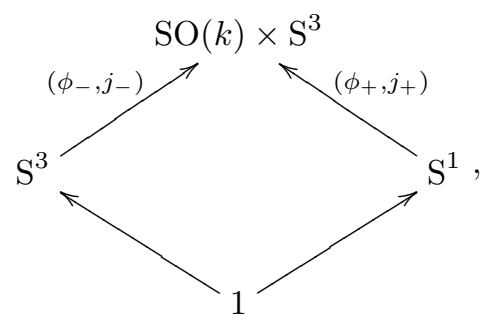

where $j_{-}=$id and $j_{+}$is the inclusion into any fixed circle subgroup of $\mathrm{S}^{3}$. The group diagram is hence determined by the homomorphisms $\phi_{-}: \mathrm{S}^{3} \rightarrow \mathrm{SO}(k)$ and $\phi_{+}: \mathrm{S}^{1} \rightarrow \mathrm{SO}(k)$. By Lemma 1.3, we can conjugate these homomorphisms $\phi_{-}$and $\phi_{+}$separately into a normal form. It is well known that $\phi_{-}$is, up to equivalence, given by a sum of irreducible representations $\phi_{1}+\cdots+\phi_{r}$, where $\phi_{i}$ either has dimension $2 n_{i}+1$ (allowing $n_{i}=0$ ) or $4 n_{i}$. By a theorem of Malcev [Ma] the image group $\phi_{-}\left(\mathrm{S}^{3}\right) \subset \mathrm{SO}(k)$ is unique up to conjugacy, unless $k$ is even. In that case one has an outer automorphism $A$ and $\phi_{-}\left(\mathrm{S}^{3}\right)$ and $A\left(\phi_{-}\left(\mathrm{S}^{3}\right)\right)$ are conjugate in $\mathrm{SO}(k)$ unless the irreducible subrepresentations are all nontrivial even dimensional. Although this change will give a cohomogeneity one manifold which, by Lemma 1.7, is not equivariantly diffeomorphic to the original one, we will see that the corresponding principal bundles are isomorphic if $k=3$ or $k \geq 5$. We define:

$$
\begin{aligned}
& m_{i}=n_{i}\left(2 n_{i}+1\right)\left(2 n_{i}+2\right) / 3 \text { if } \operatorname{dim} \phi_{i}=2 n_{i}+1, \\
& m_{i}=\left(2 n_{i}-1\right) 2 n_{i}\left(2 n_{i}+1\right) / 3 \text { if } \operatorname{dim} \phi_{i}=4 n_{i} .
\end{aligned}
$$

Furthermore, $\phi_{+}$is given by $e^{i \theta} \rightarrow \operatorname{diag}\left(R\left(q_{1} \theta\right), \ldots, R\left(q_{r} \theta\right)\right)$ if $k$ is even, and if $k$ is odd, $e^{i \theta} \rightarrow \operatorname{diag}\left(R\left(q_{1} \theta\right), \ldots, R\left(q_{r} \theta\right), 1\right)$, where $r=[k / 2]$ and $\left(q_{1}, \ldots, q_{r}\right)$ are relatively prime integers. We can now state our classification theorem of these principal bundles as follows:

Theorem 5.3. Let $P$ be the principal $\mathrm{SO}(k)$ bundle over $\mathbb{C P}^{2}$ defined by (5.2) for some integers $n_{i}$ and $q_{i}$. Assuming that $k=3$ or $k \geq 5$, we have $p_{1}=$ $\pm\left(\sum q_{i}^{2}-\sum m_{i}\right)$ and $w_{2} \equiv \sum q_{i} \bmod 2$.

Proof. We again use the decomposition $P=P_{-} \cup P_{+}$with $P_{-} \simeq \mathrm{SO}(k) \times \mathrm{S}^{3} / \mathrm{S}^{3}=$ $\mathrm{SO}(k)$ and $P_{+} \simeq \mathrm{SO}(k) \times \mathrm{S}^{3} / \mathrm{S}^{1}$ and $P_{0}=P_{-} \cap \mathrm{P}_{+} \simeq \mathrm{SO}(k) \times \mathrm{S}^{3}$ in order to apply Proposition 3.6. Notice that $\phi_{+}$is onto $\pi_{1}$, and hence $P_{+}$is simply connected, if and only if $\sum q_{i}$ is odd. Since $\pi_{1}\left(P_{-}\right)=\mathbb{Z}_{2} \rightarrow \pi_{1}\left(P_{0}\right)=\mathbb{Z}_{2}$ is an isomorphism, van Kampen implies that $P$ is simply connected, which by Proposition 3.6 means that $w_{2} \neq 0$, if and only if $\sum q_{i}$ is odd.

For the cohomology of the principal orbits we have $H^{3}\left(P_{0}\right)=H^{3}\left(\mathrm{SO}(k) \times \mathrm{S}^{3}\right)=$ $\mathbb{Z} \oplus \mathbb{Z}$. We choose a generator $x \in H^{3}(\mathrm{SO}(k))$ and $y \in H^{3}\left(\mathrm{~S}^{3}\right)$ and by abuse of notation use the same symbol for a basis in $H^{3}\left(P_{0}\right)$. The sign of these generators will be determined uniquely in the proof of Lemma 5.4. Let us first assume that $k \geq 5$.

Lemma 5.4. For $P_{+}$we have $H^{3}\left(P_{+}\right)=\mathbb{Z}$ and

(a) If $\sum q_{i}$ is odd, $H^{4}\left(P_{+}\right)=\mathbb{Z}_{2}$ and under the projection $\pi_{+}^{*}: H^{3}\left(P_{+}\right) \rightarrow$ $H^{3}\left(P_{0}\right)$ a generator goes to $-2 x+\left(\sum q_{i}^{2}\right) y$. 
(b) If $\sum q_{i}$ is even, $H^{4}\left(P_{+}\right)=\mathbb{Z}_{2} \oplus \mathbb{Z}_{2}$ and $\pi_{+}^{*}$ takes a generator to $-x+$ $\frac{1}{2}\left(\sum q_{i}^{2}\right) y$.

Proof. Let us first recall the Borel method of computing the cohomology of a homogeneous space $\mathrm{G} / \mathrm{K}$. Let $E$ be a space on which $\mathrm{G}$ acts freely, and hence $B_{\mathrm{G}}=E / \mathrm{G}$ and $B_{\mathrm{K}}=E / \mathrm{K}$ the classifying spaces for principal $\mathrm{G}$ and $\mathrm{K}$ bundles, respectively. One uses the naturality between the differentials in the following commutative diagram of $\mathrm{G}$ principal fibrations:

\section{DiAGRAM A}

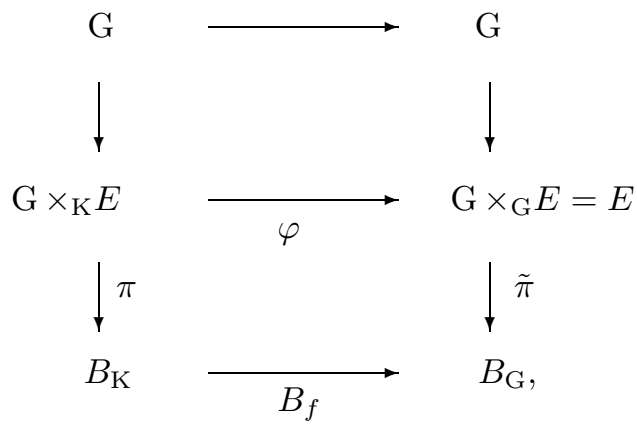

where $f: \mathrm{K} \rightarrow \mathrm{G}$ is the inclusion. The right hand side fibration is the universal $\mathrm{G}$ principal bundle. In the left hand side fibration, $\mathrm{G}$ acts freely on $\mathrm{G} \times{ }_{K} E$ via left multiplication in the first coordinate and $\pi$ is the projection onto the second coordinate. The map $B_{f}$ is therefore the classifying map of this principal bundle. The spectral sequence for the left hand side fibration computes the cohomology of $\mathrm{G} / \mathrm{K}$ since the projection onto the first coordinate $\mathrm{G} \times{ }_{\mathrm{K}} E \rightarrow \mathrm{G} / \mathrm{K}$ is a homotopy equivalence. The differentials in the spectral sequence are thus determined as soon as one computes $B_{f}^{*}$. In order to compute this map, one uses a further commutative diagram:

\section{Diagram B}

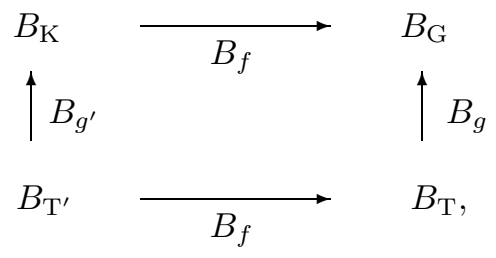

where $g^{\prime}: \mathrm{T}^{\prime} \rightarrow \mathrm{K}$ and $g: \mathrm{T} \rightarrow \mathrm{G}$ are maximal tori. The cohomology $H^{*}\left(B_{\mathrm{T}}\right)=$ $\mathbb{Z}\left[x_{1}, \ldots, x_{r}\right]$ is a polynomial ring with $\operatorname{dim} x_{i}=2$ and $r=\mathrm{rk}$. This method works well if the Lie groups involved have no torsion in cohomology since then $B_{g}^{*}$ is injective with image the Weyl group invariant elements. Extra care needs to be taken since this is not true for $\mathrm{SO}(n)$.

We now apply this to our situation, where $\mathrm{G}=\mathrm{G}_{1} \times \mathrm{G}_{2}=\mathrm{SO}(k) \times \mathrm{S}^{3}, \mathrm{~K}=\mathrm{K}^{+}=$ $\mathrm{T}^{\prime}=\mathrm{S}^{1}$ and $f=\left(f_{1}, f_{2}\right)=\left(\phi_{+}, j_{+}\right)$. By using the naturality of differentials with 
respect to the projection $\mathrm{G}_{1} \times \mathrm{G}_{2} \rightarrow \mathrm{G}_{i}$ we can break up the computation of the differentials in the left hand side spectral sequence into considering two diagrams of type $A$, one for $G=G_{1}$ and one for $G=G_{2}$. We start with the former one.

As in the proof of Proposition 3.6, we see that in the universal bundle for $\mathrm{G}_{1}$ we have that $\tilde{d}_{2}: \tilde{E}_{2}^{0,3}=H^{3}\left(\mathrm{G}_{1}, \mathbb{Z}\right)=\mathbb{Z} \rightarrow \tilde{E}_{2}^{2,2}=H^{2}\left(B_{\mathrm{G}_{1}}, H^{2}\left(\mathrm{G}_{1}\right)\right)=\mathbb{Z}_{2}$ is onto with image the second Stiefel-Whitney class. Furthermore $\tilde{d}_{4}: \tilde{E}_{4}^{0,3}=\mathbb{Z} \rightarrow \tilde{E}_{4}^{4,0}=\mathbb{Z}$ takes the generator $2 x$ to a generator which we denote by $\bar{x}$.

In order to compute $B_{f_{1}}^{*}$ let $g_{1}: \mathrm{T}^{r} \rightarrow \mathrm{SO}(k)$ be a maximal torus with coordinates $\left(s_{1}, \ldots, s_{r}\right)$. By abuse of notation we identify $s_{i} \in H^{1}\left(T^{r}\right)$ and via transgression $\bar{s}_{i} \in H^{2}\left(B_{\mathrm{T}^{r}}\right)$ and hence $H^{2}\left(B_{\mathrm{T}^{r}}\right)=\mathbb{Z}\left[\bar{s}_{s}, \ldots, \bar{s}_{r}\right]$. Similarly $u$ is a coordinate in $\mathrm{K}=\mathrm{S}^{1}$ and hence $H^{2}\left(B_{\mathrm{S}^{1}}\right)=\mathbb{Z}[\bar{u}]$. Next we claim that $B_{g_{1}}^{*}(\bar{x})=\sum \bar{s}_{i}{ }^{2}$. To see this, let $\mathrm{SO}(2)$ and $\mathrm{SO}(3)$ be the standard embeddings in $\mathrm{SO}(k)$. Since the Stiefel manifold $\mathrm{SO}(k) / \mathrm{SO}(3)$ is 2-connected with $\pi_{3}=\mathbb{Z}_{2}$, it has cohomology $H^{1}=H^{2}=H^{3}=0$ and $H^{4}=\mathbb{Z}_{2}$, and the spectral sequences of the bundle $\mathrm{SO}(k) / \mathrm{SO}(3) \rightarrow B_{\mathrm{SO}(3)} \rightarrow B_{\mathrm{SO}(k)}$ imply that $H^{4}\left(B_{\mathrm{SO}(k)}\right)=\mathbb{Z} \rightarrow H^{4}\left(B_{\mathrm{SO}(3)}\right)=$ $\mathbb{Z}$ is an isomorphism. Furthermore, the spectral sequence for $\mathrm{SO}(3) / \mathrm{SO}(2) \rightarrow$ $B_{\mathrm{SO}(2)} \rightarrow B_{\mathrm{SO}(3)}$ shows that $H^{4}\left(B_{\mathrm{SO}(3)}\right) \rightarrow H^{4}\left(B_{\mathrm{SO}(2)}\right)=\mathbb{Z}$ is an isomorphism as well. We now choose the sign of the generator $x$ and thus $\bar{x}$, so that the embedding $\mathrm{SO}(2) \rightarrow \mathrm{SO}(k)$ takes $\bar{x}$ to the square of a generator in $H^{2}\left(B_{\mathrm{SO}(2)}\right)=\mathbb{Z}$. This easily implies that $B_{g_{1}}^{*}(\bar{x})=\sum{\overline{s_{i}}}^{2}$. Since $f_{1}(u)=\left(q_{1} u, \ldots, q_{r} u\right) \in \mathrm{T}^{r}$ we have $B_{f_{1}}^{*}\left(\bar{s}_{i}\right)=q_{i} \bar{u}$ and hence $B_{f_{1}}^{*}(\bar{x})=\sum q_{i}^{2} \bar{u}^{2}$. By naturality it follows that $d_{4}(2 x)=\sum q_{i}^{2} \bar{u}^{2}$. Next we claim that $d_{2}(x) \in E_{2}^{2,2}=H^{2}\left(B_{\mathrm{S}_{1}}, H^{2}\left(\mathrm{G}_{1}\right)\right)=\mathbb{Z}_{2}$ is nonzero if and only if $\sum q_{i}$ is odd. This indeed follows since by Proposition [3.6 the $\mathrm{SO}(k)$ principal bundle $\mathrm{SO}(k) / f_{1}\left(\mathrm{~S}^{1}\right)=\mathrm{G}_{1} \times{ }_{\mathrm{S}^{1}} E \rightarrow B_{\mathrm{S}^{1}}$ is spin if and only if the total space $\mathrm{SO}(k) / f_{1}\left(\mathrm{~S}^{1}\right)$ is not simply connected.

In the universal bundle for $\mathrm{G}_{2}$ we have that $\tilde{d}_{4}: \tilde{E}_{4}^{0,3}=H^{3}\left(\mathrm{G}_{2}\right)=\mathbb{Z} \rightarrow \tilde{E}_{4}^{4,0}=$ $H^{4}\left(B_{\mathrm{G}_{2}}\right)=\mathbb{Z}$ is an isomorphism, and we denote the image of the generator $y$ by $\bar{y}$. $f_{2}: \mathrm{S}^{1} \rightarrow \mathrm{S}^{3}$ can be viewed as a maximal torus, and from the spectral sequence of $\mathrm{S}^{3} / \mathrm{S}^{1} \rightarrow B_{\mathrm{S}^{1}} \rightarrow B_{\mathrm{S}^{3}}$ it follows that we can choose the sign of $y$ such that $B_{f_{2}}^{*}(\bar{y})=\bar{u}^{2}$ and hence $d_{4}(y)=\bar{u}^{2}$.

We are now ready to consider the spectral sequence of the $G$ principal bundle $\mathrm{SO}(k) \times \mathrm{S}^{3} / \mathrm{S}^{1}=\mathrm{G} \times \mathrm{S}^{1} E \rightarrow B_{\mathrm{S}^{1}}$. The group $E_{2}^{0,3}=H^{3}(\mathrm{G})=\mathbb{Z} \oplus \mathbb{Z}$ is generated by $x, y$. Assuming that $\sum q_{i}$ is odd, we obtain by the above that $d_{2}(x) \neq 0$ and $d_{2}(y)=0$. Hence $2 x, y$ are generators of $E_{3}^{0,3}=\mathbb{Z} \oplus \mathbb{Z}$ and $d_{4}(2 x)=\sum q_{i}^{2} \bar{u}^{2}$ and $d_{4}(y)=\bar{u}^{2}$. Thus $E_{\infty}^{4,0}=E_{\infty}^{2,2}=0$ and $E_{\infty}^{0,4}=\mathbb{Z}_{2}$ and hence $H^{4}\left(P_{+}\right)=\mathbb{Z}_{2}$. Furthermore, $E_{\infty}^{0,3}=\mathbb{Z}$ with generator $-2 x+\sum q_{i}^{2} y$. This implies that $H^{3}\left(P_{+}\right)=\mathbb{Z}$ and via the edge homomorphism, $-2 x+\sum q_{i}^{2} y$ is the image of a generator in $H^{3}\left(\mathrm{G} / \mathrm{S}^{1}\right)$.

If on the other hand $\sum q_{i}$ is even, $d_{2}(x)=d_{2}(y)=0$ and hence $H^{4}\left(P_{+}\right)=$ $\mathbb{Z}_{2} \oplus \mathbb{Z}_{2}$. Now $x, y$ generate $E_{3}^{0,3}$ and $d_{4}(x)=\frac{1}{2} \sum q_{i}^{2} \bar{u}^{2}$ and $d_{4}(y)=\bar{u}^{2}$ and hence $-x+\frac{1}{2} \sum q_{i}^{2} y$ is the image of a generator. This completes the proof of Lemma 5.4

For the left half we have:

Lemma 5.5. $P_{-}$satisfies:

(a) $H^{3}\left(P_{-}\right)=\mathbb{Z}$ and $H^{4}\left(P_{-}\right)=\mathbb{Z}_{2}$ and $H^{4}\left(P_{-}\right) \rightarrow H^{4}\left(P_{0}\right)=\mathbb{Z}_{2}$ is an isomorphism. 
(b) Under the projection $\pi_{-}^{*}: H^{3}\left(P_{-}\right) \rightarrow H^{3}\left(P_{0}\right)$, a generator goes to $-x+$ $\frac{1}{2}\left(\sum m_{i}^{2}\right) y$.

Proof. We indicate the changes which are necessary. We now have $\mathrm{K}=\mathrm{K}^{-}=\mathrm{S}^{3}$ and $f=\left(f_{1}, f_{2}\right)=\left(\phi_{-}, j_{-}\right)$. If $g^{\prime}: \mathrm{S}^{1} \rightarrow \mathrm{S}^{3}$ is a maximal torus of $\mathrm{K}$ with coordinate $u$, we can identify a generator in $H^{4}\left(B_{\mathrm{K}}\right)=\mathbb{Z}$ with $\bar{u}^{2}$ via $B_{g^{\prime}}$. In the spectral sequence of $\mathrm{G} \times{ }_{\mathrm{S}^{3}} E \rightarrow B_{\mathrm{S}^{3}}$ we have that $d_{2}(x) \in E_{2}^{2,2}=0$ vanishes and hence $x, y$ generate $E_{3}^{0,3}$ with $d_{4}(y)=\bar{u}^{2}$. It remains to compute $d_{4}(x)$. For this purpose, let us assume momentarily that $f_{1}$ is an irreducible representation of dimension $2 n+1$. Standard representation theory implies that the homomorphism $f_{1}: \mathrm{S}^{1} \rightarrow$ $\mathrm{T}^{r} \subset \mathrm{SO}(k)$ takes $e^{i \theta} \in \mathrm{S}^{1}$ to $\operatorname{diag}(R(2 n \theta), R((2 n-2) \theta), \ldots, R(2 \theta)) \in \mathrm{T}^{r}$. Hence $B_{f_{1}}^{*}(\bar{x})=B_{f_{1}}^{*}\left(\sum \bar{s}_{i}^{2}\right)=2^{2}+4^{2}+\cdots+(2 n)^{2}=n(2 n+1)(2 n+2) / 3$. If on the other hand $f_{1}$ is an irreducible representation of dimension $4 n$, it can be viewed, using the usual embedding $\mathrm{SU}(2 n) \subset \mathrm{SO}(4 n)$, as a complex $2 n$-dimensional representation. Thus $f_{1}\left(e^{i \theta}\right)=\operatorname{diag}(R((2 n-1) \theta), \ldots, R(\theta), R((2 n-1) \theta), \ldots, R(\theta)) \in \mathrm{T}^{2 n}$. Notice that if we change the representation by an outer automorphism, which means that we change the embedding $\mathrm{SU}(2 n) \subset \mathrm{SO}(4 n)$, the induced homomorphism on the maximal torus is the same. Thus $B_{f_{1}}^{*}(\bar{x})=2\left(1^{2}+3^{3}+\cdots+(2 n-1)^{2}\right)=(2 n-$ 1) $2 n(2 n+1) / 3$. This process is clearly additive and we obtain $B_{f_{1}}^{*}(\bar{x})=\sum m_{i} \bar{u}^{2}$ and thus $d_{4}(x)=\frac{1}{2} \sum m_{i} \bar{u}^{2}$. As above, this finishes the proof of Lemma 5.5 .

We are now ready to combine the information in Lemma 5.4 and Lemma 5.5] in the Mayer-Vietoris sequence of $P=P_{-} \cup P_{+}$. Still assuming $k \geq 5$, we have:

$$
\begin{aligned}
0=H^{3}(P) & \rightarrow H^{3}\left(P_{-}\right) \oplus H^{3}\left(P_{+}\right)=\mathbb{Z} \oplus \mathbb{Z}^{\pi_{-}^{*}-\pi_{+}^{*} H^{3}}\left(P_{0}\right)=\mathbb{Z} \oplus \mathbb{Z} \rightarrow H^{4}(P) \rightarrow \\
& \rightarrow H^{4}\left(P_{-}\right) \oplus H^{4}\left(P_{+}\right)=H^{4}\left(P_{-}\right) \oplus \mathbb{Z}_{2} \rightarrow H^{4}\left(P_{0}\right)=\mathbb{Z}_{2} \rightarrow \cdots .
\end{aligned}
$$

From Proposition 3.6 we know that $\left|p_{1}(P)\right|=\frac{1}{2}\left|H^{4}(P)\right|$ and from Lemma 5.5 that $H^{4}\left(P_{+}\right) \rightarrow H^{4}\left(P_{0}\right)$ is an isomorphism. In the case of $w_{2} \neq 0$, we have $H^{4}\left(P_{-}\right)=\mathbb{Z}_{2}$ and thus $\left|H^{4}(P)\right|=2\left|\operatorname{cokernel}\left(\pi_{-}^{*}-\pi_{+}^{*}\right)\right|=2\left|\operatorname{det}\left(\begin{array}{cc}-1 & 2 \\ \frac{1}{2} \sum m_{i} & -\sum q_{i}^{2}\end{array}\right)\right|=2 \mid \sum q_{i}^{2}-$ $\sum m_{i} \mid$. If on the other hand $w_{2}=0$, we have $H^{4}\left(P_{-}\right)=\mathbb{Z}_{2} \oplus \mathbb{Z}_{2}$ and thus $\left|H^{4}(P)\right|=$ $4\left|\operatorname{cokernel}\left(\pi_{-}^{*}-\pi_{+}^{*}\right)\right|=4 \operatorname{det}\left|\left(\begin{array}{cc}-1 & 1 \\ \frac{1}{2} \sum m_{i} & -\frac{1}{2} \sum q_{i}^{2}\end{array}\right)\right|=2\left|\sum q_{i}^{2}-\sum m_{i}\right|$.

Finally, if $k=3$, what changes is that $H^{4}\left(P_{-}\right)=0$ and, by Poincaré duality, $H^{4}\left(P_{+}\right)=0$ if $\sum q_{i}$ is odd or $\mathbb{Z}_{2}$ if $\sum q_{i}$ is even. Since by Proposition 3.6 we now have $\left|p_{1}(P)\right|=\left|H^{4}(P)\right|$, the conclusion remains the same. This completes the proof of Theorem 5.3

For $k=3,4$, Theorem 5.3 together with Proposition 1.8 and Lemma 1.6 (c) determines which principal $\mathrm{SO}(k)$ bundles admit lifts. As remarked earlier, we were not able to determine the sign of $p_{1}$ when $k \geq 5$, which leaves an ambiguity in our classification in this case.

Since the 3-dimensional representation of $\mathrm{SU}(2)$ has $m=4$, we have in particular:

Corollary 5.6. The $\mathrm{SU}(2)$ action on $\mathbb{C P}^{2}$ with a fixed point has a lift to a principal $\mathrm{SO}(3)$ bundle if and only if $p_{1}=q^{2}$ or $p_{1}= \pm\left(q^{2}-4\right)$ for some integer $q$. 
Two of these bundles are well known in positive curvature [Sh: $q=1, p_{1}(P)=$ -3 is the Aloff-Wallach space $P=\mathrm{SU}(3) / Z(\mathrm{U}(2))$ and $q=3, p_{1}(P)=5$ the Eschenburg space $P=\operatorname{diag}\left(z, z, z^{2}\right) \backslash \mathrm{SU}(3) / \operatorname{diag}\left(1,1, z^{4}\right)$.

The same methods can be applied to the suspension action of $\mathrm{SU}(2)$ on $\mathbb{S}^{4}$, which has two fixed points. A lift of this action to a cohomogeneity one action on a principal $\mathrm{SO}(k)$ bundle has a group diagram as in (5.2) such that $\mathrm{K}^{+}=\mathrm{S}^{3}$ as well. Hence $\phi_{-}$and $\phi_{+}$are both the direct sum of irreducible representations of $\mathrm{SU}(2)$ of dimensions $2 n_{i}^{-}+1$ or $4 n_{i}^{-}$, respectively $2 n_{i}^{+}+1$ or $4 n_{i}^{+}$. The principal bundles in this case are of course all spin; i.e., they are classified by $p_{1}$. If $k>5$, Table A implies that $w_{4}$ is determined by $p_{1}$ as well. Combining Proposition 3.6 with Lemma 5.5 and the Mayer-Vietoris sequence, we obtain:

Theorem 5.7. Let $P$ be the principal $\mathrm{SO}(k)$ bundle over $\mathbb{S}^{4}$ defined by the integers $n_{i}^{-}$and $n_{i}^{+}$. Assuming that $k=3$ or $k \geq 5$, we have $p_{1}= \pm\left(\sum m_{i}^{-}-\sum m_{i}^{+}\right)$.

For bundles over $\mathbb{S}^{4}$, we can reverse the sign of $p_{1}$ by considering the pullback bundle under the antipodal map since it reverses orientation. Since it also commutes with the action of $\mathrm{SU}(2)$, it follows that if one bundle admits a lift, so does the other. This completely determines when the sum action of $\mathrm{SU}(2)$ on $\mathbb{S}^{4}$ admits a lift to a principal $\mathrm{SO}(k)$ bundle $P$. But notice that for each fixed $k$, there are only finitely many bundles that do. By Lemma 1.7 (b), the groups $G=\mathrm{U}(2)$ and $G=\mathrm{SO}(4)$ admit a lift if and only if $\mathrm{SU}(2) \subset G$ does.

In $[\mathrm{HH}]$ one also finds a classification when the action of $\mathrm{G}=\mathrm{SO}(4)$ on $\mathbb{S}^{4}$ admits a lift to a principal $\mathrm{SO}(k)$ bundle with $k=3$ and $k=4$. It is interesting to note that for $k=4$ the isomorphism type of the bundle depends on the outer automorphism group of $\mathrm{SO}(4)$. E.g. if $\phi_{-}$and $\phi_{+}$are both the standard representation of $\mathrm{K}^{ \pm} \simeq$ $\mathrm{SO}(4)$ on $\mathbb{R}^{4}$ the bundle is trivial, whereas if one changes one of these by an outer automorphism, one obtains the tangent bundle of $\mathbb{S}^{4}$.

\section{REFERENCES}

[AA] A.V. Alekseevsky and D.V. Alekseevsky, G-manifolds with one-dimensional orbit space, Ad. in Sov. Math. 8 (1992), 1-31. MR.1155662 (93c:57029)

$[\mathrm{BH}] \quad$ A. Back and W. Y. Hsiang, Equivariant geometry and Kervaire spheres, Trans. Amer. Math. Soc. 304 (1987), 207-227. MR906813 (88m:53073)

[BK1] I. Belegradek and V. Kapovitch, Topological obstructions to nonnegative curvature, Math. Ann. 320 (2001), 167-190. MR1835067 (2002d:53044)

[BK2] I. Belegradek and V. Kapovitch, Obstructions to nonnegative curvature and rational homotopy theory, J. Amer. Math. Soc. 16 (2003), 259-284 . MR.1949160 (2003i:53043)

[Br] G. E. Bredon, Introduction to compact transformation groups, Academic Press, New York, 1972, Pure and Applied Mathematics, Vol. 46. MR0413144 (54:1265)

[CG] J. Cheeger and D. Gromoll, On the structure of complete manifolds of nonnegative curvature, Ann. of Math. (2) 96 (1972), 413-443. MR0309010(46:8121)

[DR] A. Derdzinski and A. Rigas. Unflat connections in 3-sphere bundles over $\mathbb{S}^{4}$,Trans. Amer. Math. Soc., 265 (1981), 485-493. MR610960 (82i:53026)

[DW] A. Dold and H. Whitney, Classification of oriented sphere bundles over a 4-complex, Ann. of Math. (2) 69 (1959), 667-677. MR0123331 (23:A659)

[EZ] C. Escher and W. Ziller, Topology of non-negatively curved manifolds, in preparation.

[FU] D. Freed and K. Uhlenbeck, Instantons and four-manifolds, Springer-Verlag, New York, 1984. MR 757358 (86c:57031)

[GZ] K. Grove and W. Ziller, Curvature and symmetry of Milnor spheres, Ann. of Math. (2) 152 (2000), 331-367. MR1792298 (2001i:53047)

[GWZ] K. Grove, B. Wilking and W. Ziller, Positively curved cohomogeneity one manifolds and 3-Sasakian geometry, J. Diff. Geom. 78 (2008), 33-111. MR2406265 (2009m:53090) 
[HH] I. Hambleton and J. C. Hausmann, Equivariant principal bundles over spheres and cohomogeneity one manifolds, Proc. London Math. Soc. 86 (2003), 250-272. MR.1971468 (2004h:55014)

[Ha] R. Hamilton, Four-manifolds with positive curvature operator, J. Diff. Geom. 24 (1986), 153-179. MR862046 (87m:53055)

[HY] A. Hattori and T. Yoshida, Lifting compact group actions in fiber bundles, Japan. J. Math. 2 (1976) 13-25. MR0461538 (57:1523)

[Ho] C. Hoelscher, Cohomogeneity one manifolds in low dimensions, Ph.D. thesis, University of Pennsylvania, 2007.

[HHs] W. C. Hsiang and W. Y. Hsiang, On compact subgroups of the diffeomorphism groups of Kervaire spheres, Ann. of Math. (2) 85 (1967), 359-369. MR0214083 (35:4934)

[La] R. K. Lashof, Equivariant bundles, Illinois J. Math. 26 (1982), 257-271. MR650393 (83g:57025)

[Ma] A. Malcev, On semi-simple subgroups of Lie groups, Izv. Akad. Nauk SSSR 8 (1944), 143174. (Russian); Amer. Math. Soc. Transl. 33 (1950). MR0011303 (6:146b) MR0037848 $(12: 317 \mathrm{c})$

[Mi] J. Milnor and J. D. Stasheff, Characteristic classes, Princeton University Press, Princeton, N. J., 1974, Annals of Mathematics Studies, No. 76. MR0440554 (55:13428)

[Mo] P. Mostert, On a compact Lie group acting on a manifold, Ann. of Math. (2) 65 (1957), 447-455; Errata, On a compact Lie group acting on a manifold, Ann. of Math. (2) 66 (1957), 589. MR0085460(19:44b) MR0095897 (20:2395)

[Ne] W. Neuman, 3-dimensional G-manifolds with two-dimensional orbits, in: P. S. Mostert (ed.), Proceedings of Conference on Transformation Groups, Springer-Verlag (1968), 220222. MR0245043(39:6355)

[OSS] C. Okonek, M. Schneider and H. Spindler, Vector bundles on compex projective spaces, Birkhäuser 1980, Progress in Math. Vol 3. MR.561910 (81b:14001)

[OW] M. Özaydin and G. Walschap, Vector bundles with no soul, Proc. Amer. Math. Soc. 120 (1994), 565-567. MR:1162091(94d:53057)

[Pa] J. Parker, 4-dimensional G-manifolds with 3-dimensional orbits, Pacific J. Math. 125 (1986), 187-204. MR860758 (88e:57033)

[PS] R. S. Palais and T. E. Stewart, The cohomology of differentiable transformation groups, Amer. J. Math. 83 (1961), 623-644. MR.0140613 (25:4030)

[Ri] A. Rigas, Geodesic spheres as generators of the homotopy groups of $O, B O$, J. Diff. Geom. 13 (1978), 527-545. MR.570216 (81e:57043)

[Sh] K. Shankar, On the fundamental groups of positively curved manifolds, J. Differential Geom. 49 (1998), 179-182. MR.1642117 (99h:53040)

[St] T. E. Stewart, Lifting group actions in fibre bundles, Ann. of Math. (2) $\mathbf{7 4}$ (1961), 192198. MR0126502 (23:A3798)

[Ta] B. Tataro, Cheeger Manifolds and the Classification of Biquotients, J. Diff. Geom. 61 (2002), 397-451. MR1979366 (2004b:53075)

[TD] T. Tom Diek, Faserbündel mit Gruppenoperationen, Arch. Math. 20 (1969), 136-143. MR 0245027 (39:6340)

[Ya] D. Yang, On complete metrics of nonnegative curvature on 2-plane bundles, Pacific J. Math. 171 (1995), 569-583. MR.1372245 (96k:53034)

Department of Mathematics, University of Maryland, College Park, Maryland 20742 E-mail address: kng@math.umd.edu

Current address: Department of Mathematics, University of Notre Dame, 255 Hurley Hall, Notre Dame, Indiana 46556-4618

E-mail address: kgrove2@nd.edu

Department of Mathematics, University of Pennsylvania, Philadelphia, Pennsylvania 19104

E-mail address: wziller@math.upenn.edu 